\title{
Research on Nonlinear Coupled Tracking Controller for Double Pendulum Gantry Cranes With Load Hoisting/lowering
}

\section{Huaitao Shi}

Shenyang Jianzhu University https://orcid.org/0000-0002-1574-5633

\section{Fuxing Yao}

Shenyang Jianzhu University

Zhe Yuan ( $\nabla$ yuanzhe@sjzu.edu.cn )

Shenyang Jianzhu University https://orcid.org/0000-0002-4490-9908

Shenghao Tong

Shenyang Jianzhu University

\section{Yinghan Tang}

University of California Riverside Bourns College of Engineering

\section{Gang Han}

shenyang yadong technology co., Itd

\section{Research Article}

Keywords: underactuated crane, double pendulum, nonlinear anti-swing control, Lyapunov techniques, load hoisting and lowering, tracking control

Posted Date: February 23rd, 2021

DOl: https://doi.org/10.21203/rs.3.rs-224720/v1

License: (c) (1) This work is licensed under a Creative Commons Attribution 4.0 International License. Read Full License

Version of Record: A version of this preprint was published at Nonlinear Dynamics on January 17th, 2022. See the published version at https://doi.org/10.1007/s11071-021-07185-6. 


\title{
Research on nonlinear coupled tracking controller for double pendulum gantry cranes with load hoisting/lowering
}

\author{
Huaitao Shi • Fuxing Yao • Zhe Yuan • \\ Shenghao Tong · Yinghan Tang • Gang Han
}

Received: date / Accepted: date

\begin{abstract}
Gantry cranes, which have attracted extensive attention, are mostly simplified as nonlinear single pendulum systems without load hoisting/lowering. However, in fact, due to the existence of the hook, gantry cranes produce double pendulum swing. With an extra underactuated degree of freedom, the antiswing control of the double pendulum gantry cranes becomes more difficult than that of single pendulum gantry cranes, especially when load hoisting/lowering is considered simultaneously. Moreover, large swings, which lead to problems such as inaccurate positioning and low transportation efficiency, may be caused by double pendulum gantry cranes with load hoisting/lowering. In this paper, a nonlinear coupled tracking anti-swing controller is proposed to solve these problems. In this controller, a smooth tracking trajectory is introduced to ensure the stable start and run of the trolley, and a coupled signal is constructed to eliminate the residual swing angles of gantry crane system. The system stability is analyzed by using Lyapunov method and Barbarat theorem. Theoretical derivation, simulation and experimental results show that the proposed controller has excellent control performance, specifically, not only does it ensure accurate positioning of the load, but also it suppresses and eliminates the hook/load swing angle effectively. The proposed controller can still
\end{abstract}

H. Shi $\cdot$ F. Yao $\cdot$ Z. Yuan $(\bowtie) \cdot$ S. Tong

School of Mechanical Engineering, Shenyang Jianzhu University, Shenyang 110168, China

E-mail: yuanzhe@sjzu.edu.cn

\section{Y. Tang}

Bourns College of Engineering, University of California Riverside, Los Angeles 90001, United States

G. Han

Shenyang Yadong Technology Co., Ltd, Shenyang 110000, China achieve good control effects and has strong robustness under the condition of changing the load mass, trolley target displacement, system initial swing angles and adding external disturbance.

Keywords underactuated crane - double pendulum . nonlinear anti-swing control · Lyapunov techniques · load hoisting and lowering · tracking control

\section{Introduction}

Underactuated gantry cranes, as a kind of large-scale lifting facilities, have the characteristics of flexible system, efficient transportation and low cost, so gantry cranes are widely used in many industries such as construction, metallurgy and logistics $[1,2]$. As a representative nonlinear underactuated system, the number of independent input control variables of gantry crane is less than the number of system degrees of freedom [3]. The goals of gantry crane are not only to move the load to the target position accurately, but also to suppress and eliminate the system swings effectively [4]. In fact, it is easy to produce large swing angles of the system when the trolley moves rapidly or encounters external interference (wind load, impact, etc.), which affect the load accurate positioning, and even cause serious safety accidents. In some special working conditions, such as automatic driving of gantry cranes, the linkage control of the load hoisting/lowering motion and trolley horizontal motion improves the transport efficiency of gantry cranes. Compared with single control, the linkage control greatly improves the working efficiency of gantry cranes, and is worthy of in-depth study. Moreover, gantry crane may produce double pendulum swing when the hook mass or the rope length between the 
load and hook cannot be ignored [5]. Under this condition, the anti-swing controllers of single pendulum cranes are difficult to be applied to double pendulum cranes. Hence, it is not of great significance to research on anti-swing controllers of single pendulum cranes. If it is operated manually, not only is the working efficiency low, but also the trolley positioning is not accurate, and it is easy to cause large hook/load swing angle, and poor safety. Therefore, a large number of scholars are committed to the research on anti-swing controller of double pendulum underactuated gantry crane (DPUGC).

DPUGC, however, has an extra underactuated degree of freedom, which makes it more challenging to establish dynamic model and design corresponding antiswing controller. Moreover, although the linkage control makes the design of the anti-swing controller difficult, it is more in line with the actual conditions and is worthy of in-depth research. Most existing researches simplify crane model for convenience, so as to research on the anti-swing method of nonlinear single pendulum cranes, such as trajectory planning controller [6], nonlinear feedback controller [7-11], while the researches based on DPUGC are still in their infancy [5]. At present, in-put shaping controller is the most widely used strategy for DPUGC [12]. However, in this strategy, many parts of linearization exist in the dynamic model and controller, which lead to some errors inevitably. Tuan et al. propose a sliding mode control method [13], which can improve the trolley positioning accuracy and suppress the system swings effectively, but some nonlinear variables are ignored when they analyze system stability. In reference [14], sun et al. propose a super-twisting-based anti-swing control method that ensures the trolley positioning and the suppresses system swings by using a linear model. Qian et al. propose an input shaping anti-swing control method to avoid complex analysis of crane dynamic model [15]. Although the above references [12-15] do more or less linearization in the crane dynamic model or controller design process, they can play a certain controlling role in suppressing and eliminating the swing angles. The time-optimal controller and energy-optimal controller are proposed in reference [16] and [17] respectively. However, the above two controllers require a large amount of offline calculation, and cannot consider the linkage control of the load hoisting/lowering motion and trolley horizontal motion. Zhang et al. propose an online trolley motion planning method [18], which can suppress and eliminate the system swing angles effectively while ensuring the trolley precise positioning, but partial approximations are applied to the process of dynamic analysis, and the load hoisting/lowering mo- tion is not considered. A saturation controller is proposed in reference [19], but the load hoisting/lowering motion is not considered. An adaptive method is proposed in reference [5], which is one of the few research results that consider double-pendulum and load hoisting/lowering motion simultaneously. Unfortunately, the load and hook still have small repeated oscillations after the trolley stops moving, which affects safety seriously.

With the continuous exploration of relevant scholars, various anti-swing controllers have been developed. However, there are still some core issues to be resolved, mainly including the following shortcomings: 1) Most existing methods are developed based on open-loop control, and their robustness is poor. Once the system suffers from external disturbance or the system parameters are changed, the control effect may be affected; 2) Partial linearization or approximations exist in dynamic analysis and controller design in many researches, which have insufficient accuracy; 3) For DPUGC, most existing achievements do not consider the load hoisting/lowering motion, however, in practice, linkage control, which is more challenging than single control, is inevitable to be considered; 4) In addition, many researches do not consider the impact of the swing angles, especially the coupling of the trolley displacemen$\mathrm{t}$ information, rope length information and swing angles information is necessary when considering the load hoisting/lowering.

Inspired by previous researches on underactuated crane system [1,3], an nonlinear coupled tracking antiswing control method for DPUGC with load hoisting motion is proposed. The proposed method ensures smooth start and stable operation of the trolley by introducing a smooth reference trajectory; a coupling signal, which includes the information of the load/hook swing angle, trolley and rope, is introduced into the controller to suppress and eliminate the system swings effectively. Then, the system stability is strictly analyzed by using Lyapunov method and Barbarat theorem. Finally, the actual effectiveness of the proposed controller is proved by comparing simulations, experiments and robustness verification simulations; especially good control results and strong robustness can still be obtained when changing the trolley target displacement, the load mass, the initial hook/load swing angle, and adding external disturbance. The main contributions of this paper are as follows:

1) Not only does the proposed controller consider double pendulum effect, but also it considers the linkage of the trolley motion and load lifting motion, and it does not need to linearize or approximate the dynamics and controller of gantry crane. 
2) The proposed controller ensures stable start and stable operation of the trolley, and the system repeated swings are eliminated.

3) The proposed controller has strong robustness under the condition of changing the load mass, trolley target displacement, load/hook initial swing angle and adding external disturbance.

The other parts of this paper are stated as follows: the nonlinear dynamic model of DPUGC with load hoisting and lowering is established in Section 2; the antiswing controller design and stability analysis is performed in Section 3; the feasibility of the proposed controller is verified by a large number of simulations and experiments in Section 4; the conclusion is described in Section 5 .

\section{Nonlinear dynamic model of DPUGC with load hoisting/lowering}

The DPUGC linkage system is described in Figure 1. $M$ refers to the trolley mass, $m_{1}$ refers to the hook mass, $m_{2}$ refers to the load mass; $x(t)$ refers to the displacement of the trolley; $l_{1}(t)$ refers to the rope length; $l_{2}$ denotes the distance of the hook from the load; $\theta_{1}(t)$ denotes the hook swing angle; $\theta_{2}(t)$ refers to the load swing angle; The horizontal driving force $F_{x}(t)$ drives the trolley to slide on the track, and the driving force $F_{l}(t)$ changes the rope length $l_{1}(t)$ to lift the load, they can ensure that the load reaches the target position quickly.

Remark 1 Double pendulum and load hoisting/lowering motion need to be considered when establishing crane dynamics model and designing anti-swing controller, but there are only a handful of articles considering these two cases. In practice, for improving the transportation efficiency, the trolley motion and load hoisting/lowering motion are usually combined; When the hook mass and the rope length cannot be ignored, the double pendulum effect will occur. From the practical application, the DPUGC anti-swing controller is more worthy of research than the single pendulum crane.

The Euler Lagrange equation is adopted to build the nonlinear dynamic model of the DPUGC linkage system $[1,5]$ :

$$
\begin{aligned}
& {\left[\left(\ddot{l}_{1}-l_{1} \dot{\theta}_{1}^{2}\right) \sin \theta_{1}+\left(l_{1} \ddot{\theta}_{1}+2 \dot{\theta}_{1} \dot{l}_{1}\right) \cos \theta_{1}\right]} \\
& \cdot\left(m_{1}+m_{2}\right)+m_{2} l_{2}\left(\ddot{\theta}_{2} \cos \theta_{2}-\dot{\theta}_{2}^{2} \sin \theta_{2}\right) \\
& +\left(M+m_{1}+m_{2}\right) \ddot{x}=F_{x}-F_{r x}-d_{x} \dot{x} \\
& \left(m_{1}+m_{2}\right)\left[\sin \theta_{1} \ddot{x}+\ddot{l}_{1}-l_{1} \dot{\theta}_{1}^{2}-g \cos \theta_{1}\right] \\
& +m_{2} l_{2}\left(\sin \left(\theta_{1}-\theta_{2}\right) \ddot{\theta}_{2}-\cos \left(\theta_{1}-\theta_{2}\right) \dot{\theta}_{2}^{2}\right) \\
& =F_{l}-d_{l} \dot{l}
\end{aligned}
$$

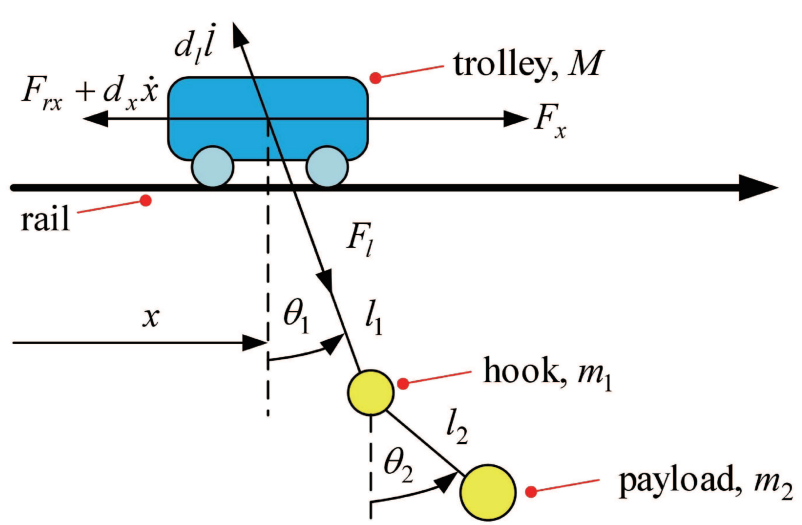

Fig. 1 DPUGC linkage system

$$
\begin{aligned}
& \left(m_{1}+m_{2}\right)\left(l_{1} \cos \theta_{1} \ddot{x}+l_{1}^{2} \ddot{\theta}_{1}+2 l_{1} \dot{\theta}_{1} \dot{l}_{1}+g l_{1} \sin \theta_{1}\right) \\
& +m_{2} l_{1} l_{2}\left[\cos \left(\theta_{1}-\theta_{2}\right) \ddot{\theta}_{2}+\sin \left(\theta_{1}-\theta_{2}\right) \dot{\theta}_{2}^{2}\right] \\
& =-d_{\theta_{1}} \dot{\theta}_{1} \\
& m_{2} l_{2}\left[\begin{array}{l}
\cos \theta_{2} \ddot{x}+\sin \left(\theta_{1}-\theta_{2}\right)\left(\ddot{l}_{1}-l_{1} \dot{\theta}_{1}^{2}\right)+l_{2} \ddot{\theta}_{2} \\
+\cos \left(\theta_{1}-\theta_{2}\right)\left(l_{1} \ddot{\theta}_{1}+2 \dot{\theta}_{1} \dot{l}_{1}\right)+g \sin \theta_{2}
\end{array}\right] \\
& =-d_{\theta_{2}} \dot{\theta}_{2}
\end{aligned}
$$

Where $d_{x} \in \mathbf{R}^{+}$is the coefficient of air resistance on the trolley; $d_{l} \in \mathbf{R}^{+}$is the coefficient of mechanical friction on the rope; $g$ is the acceleration of gravity; $d_{\theta_{1}}, d_{\theta_{2}}$ are the coefficients of air resistance. $F_{r x} \in \mathbf{R}^{+}$ is the friction force between trolley and track, and its definition formula is [20-23]:

$F_{r x}=f_{r 0 x} \tanh \left(\frac{\dot{x}}{\varepsilon_{x}}\right)+k_{r x}|\dot{x}| \dot{x}$

Where $f_{r 0 x}, \varepsilon_{x} \in \mathbf{R}^{+}$are coefficients related to static friction, and $k_{r x} \in \mathbf{R}^{+}$is coefficient related to viscous friction.

Remark 2 In the nonlinear dynamic model (1)-(4) of the DPUGC, external factors, such as air resistance and mechanical friction, have been covered, and the model has not been linearized or approximated, which makes the model more exact.

The nonlinear dynamic model is rewritten for the convenience of subsequent controller development and stability analysis:

$$
\mathbf{M}(\mathbf{q}) \ddot{\mathbf{q}}+\mathbf{V}(\mathbf{q}, \dot{\mathbf{q}}) \dot{\mathbf{q}}+\mathbf{G}(\mathbf{q})=\mathbf{U}+\mathbf{F}_{d}
$$

Where $\mathbf{q}(t)=\left[x(t), l_{1}(t), \theta_{1}(t), \theta_{2}(t)\right]^{\mathrm{T}}$ represents the system state vector, $\mathbf{M}(\mathbf{q}), \mathbf{V}(\mathbf{q}, \dot{\mathbf{q}}) \in \mathbf{R}^{4 \times 4}$ are the system inertia matrix and the system centripetal-Coriolis force matrix, $\mathbf{G}(\mathbf{q}) \in \mathbf{R}^{4}, \mathbf{U}$ and $\mathbf{F}_{\mathbf{d}} \in \mathbf{R}^{4}$ represent the system gravitational potential energy vector, the system drive vector and the system disturbance vector 
respectively. Their detailed forms are as follows:

$\mathbf{M}=\left[\begin{array}{cccc}a_{11} & a_{12} & a_{13} & a_{14} \\ a_{12} & a_{22} & 0 & a_{24} \\ a_{13} & 0 & a_{33} & a_{34} \\ a_{14} & a_{24} & a_{34} & a_{44}\end{array}\right], \mathbf{V}=\left[\begin{array}{cccc}0 & b_{12} & b_{13} & b_{14} \\ 0 & 0 & b_{23} & b_{24} \\ 0 & -b_{23} & b_{33} & b_{34} \\ 0 & b_{42} & b_{43} & 0\end{array}\right]$,

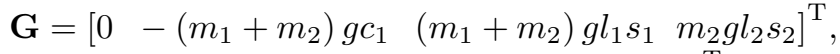

$\mathbf{F}_{d}=\left[\begin{array}{llll}-F_{r x}-d_{x} \dot{x} & -d_{l} \dot{l}_{1} & -d_{\theta_{1}} \dot{\theta}_{1} & -d_{\theta_{2}} \dot{\theta}_{2}\end{array}\right]^{\mathrm{T}}$,

$\mathbf{U}=\left[\begin{array}{llll}F_{x} & F_{l} & 0 & 0\end{array}\right]^{\mathrm{T}}$,

Wherein $a_{11}=M+m_{1}+m_{2}, a_{12}=\left(m_{1}+m_{2}\right) s_{1}, a_{13}=$ $\left(m_{1}+m_{2}\right) l_{1} c_{1}, a_{14}=m_{2} l_{2} c_{2}, a_{22}=m_{1}+m_{2}, a_{24}=$ $m_{2} l_{2} s_{1-2}, a_{33}=\left(m_{1}+m_{2}\right) l_{1}^{2}, a_{34}=m_{2} l_{1} l_{2} c_{1-2}, a_{44}=$ $m_{2} l_{2}^{2}, b_{12}=\left(m_{1}+m_{2}\right) \dot{\theta}_{1} c_{1}, b_{13}=\left(m_{1}+m_{2}\right) \dot{l}_{1} c_{1}-$ $\left(m_{1}+m_{2}\right) l_{1} s_{1} \dot{\theta}_{1}, b_{14}=-m_{2} l_{2} s_{2} \dot{\theta}_{2}, b_{23}=-\left(m_{1}+m_{2}\right)$. $l_{1} \dot{\theta}_{1}, b_{24}=-m_{2} l_{2} c_{1-2} \dot{\theta}_{2}, b_{33}=\left(m_{1}+m_{2}\right) l_{1} \dot{l}_{1}, b_{34}=$ $m_{2} l_{1} l_{2} s_{1-2} \dot{\theta}_{2}, b_{42}=m_{2} l_{2} c_{1-2} \dot{\theta}_{1}, b_{43}=m_{2} l_{2} \dot{l}_{1} c_{1-2}-$ $m_{2} l_{1} l_{2} \dot{\theta}_{1} s_{1-2}$. The above variables are expressed as follows:

$s_{i}=\sin \theta_{i}, c_{i}=\cos \theta_{i}, s_{i \pm j}=\sin \left(\theta_{i} \pm \theta_{j}\right)$,

$c_{i \pm j}=\cos \left(\theta_{i} \pm \theta_{j}\right), i, j=1,2(i \neq j)$

The DPUGC linkage system has the following properties:

Property 1 Matrix $\mathbf{M}(\mathbf{q})$ is always a positive definite symmetric matrix.

Property 2 The relationship between matrix $\mathbf{M}(\mathbf{q})$ and $\mathbf{V}(\mathbf{q}, \dot{\mathbf{q}})$ matrix is as follows:

$\mathbf{y}^{\mathrm{T}}\left[\frac{1}{2} \dot{\mathbf{M}}(\mathbf{q})-\mathbf{V}(\mathbf{q}, \dot{\mathbf{q}})\right] \mathbf{y}=0 \quad \forall \mathbf{y} \in \mathbf{R}^{4}$

According to the actual engineering situation of gantry crane linkage system, the assumptions are as follows:

Assumption 1 Ignore the mass and flexibility of the rope.

Assumption 2 In the actual working process, the load is below the trolley, and the hook/load swing angle should meet the following conditions [24,25]:

Assumption 3 Both the initial length $l_{1}(0)$ and target length $p_{d l}$ of the rope are not longer than the maximum allowable length $L$ of the crane:

$0<l_{1}(0), l_{1}, p_{d l} \leq L$

Assumption 4 In practice, the driving force of the motor is limited. On the basis of Newton's second law, the accelerations of the trolley and rope are also bounded: $0 \leq\left|F_{x}\right| \leq \bar{F}, 0 \leq|\ddot{x}| \leq \bar{a}_{x}, 0 \leq\left|F_{l}\right| \leq \bar{F}_{l}, 0 \leq\left|\ddot{l_{1}}\right| \leq \bar{a}_{l}$

The control objectives of the DPUGC linkage system are as follows [24,25]:

(1) The trolley is driven from the initial position $x(0)$ to the target position $p_{d x}$, and drives the hook from the initial length $l_{1}(0)$ to the target length $p_{d l}$.
(2) In the working process, the swing angles $\theta_{1}(t)$, $\theta_{2}(t)$ shall be suppressed and eliminated.

$\lim _{t \rightarrow \infty}\left[\begin{array}{llll}x(t) & l_{1}(t) & \theta_{1}(t) & \theta_{2}(t)\end{array}\right]^{\mathrm{T}}=\left[\begin{array}{llll}p_{d x} & p_{d l} & 0 & 0\end{array}\right]^{\mathrm{T}}$

\section{Main Results}

In this section, for improving the control performance, a nonlinear coupled tracking controller is proposed. Then, Lyapunov method and Barbarat theorem are used to perform stability analysis. The DPUGC control system is described in Figure 2.

\subsection{Controller design}

After the physical analysis of DPUGC, its energy can be deduced:

$$
\begin{gathered}
E(t)=\frac{1}{2} \dot{\mathbf{q}}^{\mathrm{T}} \mathbf{M}(\mathbf{q}) \dot{\mathbf{q}}+m_{2} g l_{2}\left(1-c_{2}\right) \\
+\left(m_{1}+m_{2}\right) g l_{1}\left(1-c_{1}\right)
\end{gathered}
$$

In view of the relationship between matrix $\mathbf{M}(\mathbf{q})$ and matrix $\mathbf{V}(\mathbf{q}, \dot{\mathbf{q}})$ in property 2 , it can be inferred that:

$\dot{\mathbf{q}}^{\mathrm{T}}\left[\mathbf{M}(\mathbf{q}) \ddot{\mathbf{q}}+\frac{1}{2} \dot{\mathbf{M}}(\mathbf{q}) \dot{\mathbf{q}}\right]=\dot{\mathbf{q}}^{\mathrm{T}}\left[\mathbf{U}+\mathbf{F}_{d}-\mathbf{G}(\mathbf{q})\right]$

Calculating the first derivative of the energy function shown in equation (9), and substituting equation (10) into the equation, we can infer:

$$
\begin{aligned}
\dot{E}(t)= & \dot{\mathbf{q}}^{\mathrm{T}}\left(\mathbf{M}(\mathbf{q}) \ddot{\mathbf{q}}+\frac{1}{2} \dot{\mathbf{M}}(\mathbf{q}) \dot{\mathbf{q}}\right)+m_{2} g l_{2} \dot{\theta}_{2} s_{2} \\
& +\left(m_{1}+m_{2}\right) g\left(i_{1}-i_{1} c_{1}+l_{1} \dot{\theta}_{1} s_{1}\right) \\
= & \dot{l}_{1}\left[F_{l}-d_{l} \dot{l}+\left(m_{1}+m_{2}\right) g\right] \\
& +\dot{x}\left(F_{x}-F_{r x}-d_{x} \dot{x}\right)-d_{\theta_{1}} \dot{\theta}_{1}^{2}-d_{\theta_{2}} \dot{\theta}_{2}^{2}
\end{aligned}
$$

The linkage system has four underactuated variables, but only two control variables. Therefore, the suppression and elimination of swing angles need to be realized by the motion of the trolley and rope. However, it is difficult to add the factors related to the hook/load swing into the controller directly while ensuring the system stability. Moreover, the trolley cannot respond to the swing angles timely and effectively when the controller contains insufficient feedback signal of swing angles, which will make it difficult to obtain excellent transient control performance and strong robustness.

To solve this problem, a generalized horizontal displacement signal, which includes the trolley displacement and swing angles, is constructed:

$\chi=x-k_{1} l_{1} \int_{0}^{t} \theta_{1} d t-k_{2} l_{2} \int_{0}^{t} \theta_{2} d t$ 


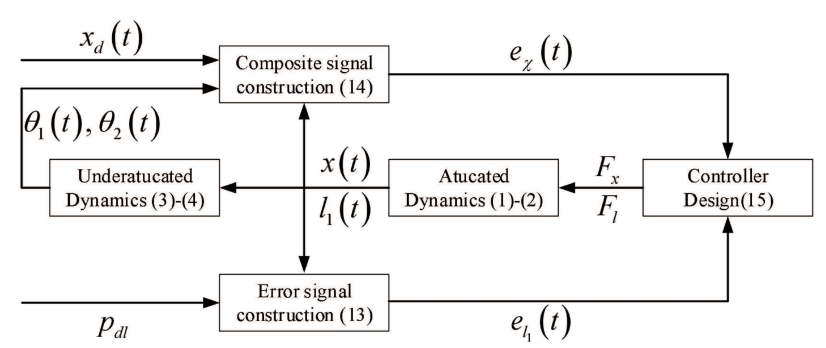

Fig. 2 DPUGC control system

Where $k_{1} \in \mathrm{R}^{+}, k_{2}=\frac{m_{2}}{m_{1}+m_{2}} k_{1}$ represent positive gains. Furthermore, for facilitating controller design, an error signal vector is defined as follows:

$\left[\begin{array}{llll}e_{x} & e_{l_{1}} & e_{\theta_{1}} & e_{\theta_{2}}\end{array}\right]^{T}=\left[\begin{array}{llll}x-x_{r} & l_{1}-p_{d l} & \theta_{1} & \theta_{2}\end{array}\right]^{\mathrm{T}}$

Where $x_{r}$ represents an expected trajectory to ensure the smooth operation of the trolley. The determination should meet the following principle [26].

Principle 1 The expected trajectory $x_{r}$ is bounded, and the trolley is guaranteed to converge from the initial displacement $x_{r}(0)$ to the target displacement $p_{d x}$ over time; The first derivative $\dot{x}_{r}$, second derivative $\ddot{x}_{r}$ and third derivative $x_{r}{ }^{(3)}$ of the expected trajectory $x_{r}$ are bounded, and gradually converge to 0 over time. They can be described as:

$\left|x_{r}(t)\right| \leq p_{d x}, 0 \leq \dot{x}_{r}(t) \leq v_{x \max },\left|\ddot{x}_{r}(t)\right| \leq a_{x \max }$, $\left|x_{r}{ }^{(3)}(t)\right| \leq j_{x \max }$

Where $v_{x \max }, a_{x \max }, j_{x \max }$ and are respectively the upper limit value of velocity, acceleration and plus acceleration.

In view of equation (12) and equation (13), a generalized horizontal trajectory tracking error signal is defined as:

$e_{\chi}=\chi-x_{r}=x-x_{c}$

Where $x_{c}$ is a simple expression for $x_{r}+k_{1} l_{1} \int_{0}^{t} \theta_{1} d t+$ $k_{2} l_{2} \int_{0}^{t} \theta_{2} d t$.

On this basis, a closed-loop feedback controller can be designed for the DPUGC linkage system as follows:

$$
\left\{\begin{array}{c}
F_{x}=F_{r x}+d_{x} \dot{x}-k_{p x} e_{\chi}-k_{d x} \dot{e}_{\chi} \\
+\left(M+m_{1}+m_{2}\right) \ddot{x}_{c} \\
F_{l}=d_{l} \dot{l}_{1}-k_{p l} e_{l_{1}}-k_{d l} \dot{e}_{l_{1}} \\
-\left(m_{1}+m_{2}\right)\left(g-\ddot{x}_{c} s_{1}\right)
\end{array}\right.
$$

Where $k_{p x}, k_{d x}, k_{p l}, k_{d l} \in \mathrm{R}^{+}$represent positive control gains to be adjusted.

Remark 3 The design of the controller $F_{x}$ mainly depends on the applied smooth trajectory $x_{r}$ and constructed coupling signal $\chi$. The smooth tracking trajectory $x_{r}$ can ensure the smooth start and stable operation of the trolley, but the swing angles (i.e., $\left.\theta_{1}, \theta_{2}\right)$ are large and in a state of repeated swings, which are difficult to suppress and eliminate. However, the designed coupling signal $\chi$ can solve this problem and realize precise positioning and rapid swing elimination while ensuring stable operation. Among them, the first derivative of the applied trajectory should meet $\dot{x}_{r}(t) \geq 0$, which is to avoid the back-and-forth motion of the trolley, reduce the wear of components and improve the working efficiency.

\subsection{Stability analysis}

Theorem 1 The nonlinear coupled tracking controller ensures that the trolley can move to the target position $p_{d x}$ accurately and the rope can reach the target length $p_{d l}$ quickly, while suppressing and eliminating the system swings effectively, which can be stated as:

$\lim _{t \rightarrow \infty}\left[\begin{array}{llllllll}x(t) & \dot{x}(t) & l_{1}(t) & i_{1}(t) & \theta_{1}(t) & \dot{\theta}_{1}(t) & \theta_{2}(t) & \dot{\theta}_{2}(t)\end{array}\right]^{\mathrm{T}}$ $=\left[\begin{array}{llllllll}p_{d x} & 0 & p_{d l} & 0 & 0 & 0 & 0 & 0\end{array}\right]^{\mathrm{T}}$

Proof The tracking error signal vector e $(t)$ is constructed as follows:

$\mathbf{e}(t)=\mathbf{q}-\mathbf{q}_{d}=\left[\begin{array}{llll}e_{\chi} & e_{l_{1}} & \theta_{1} & \theta_{2}\end{array}\right]^{\mathrm{T}}$

Where $\mathbf{q}_{d}=\left[\begin{array}{llll}x_{c} & 0 & 0 & 0\end{array}\right]^{\mathrm{T}}$.

Inspired by Equation (9), an energy-like function $E_{d}(t)$ is designed as follows:

$$
\begin{aligned}
E_{d}(t)= & \frac{1}{2} \dot{\mathbf{e}}^{\mathrm{T}} \mathbf{M}(\mathbf{q}) \dot{\mathbf{e}}+m_{2} g l_{2}\left(1-\cos \theta_{2}\right) \\
& +\left(m_{1}+m_{2}\right) g l_{1}\left(1-\cos \theta_{1}\right)
\end{aligned}
$$

In order to prove the DPUGC linkage system stability, and in view of the basis of equation (17), a positive scalar function $V(t)$ is defined as the Lyapunov candidate function as follows:

$V(t)=E_{d}(t)+\frac{1}{2} k_{p x} e_{\chi}^{2}+\frac{1}{2} k_{p l} e_{l_{1}}{ }^{2}$

Equations (6), (16)-(17) are substituted into the first derivative of equation (18), we can infer:

$$
\begin{aligned}
\dot{V}(t)= & \dot{\mathbf{e}}^{\mathrm{T}}\left[\mathbf{M}(\mathbf{q}) \ddot{\mathbf{e}}+\frac{1}{2} \dot{\mathbf{M}}(\mathbf{q}) \dot{\mathbf{e}}\right]+k_{p x} e_{\chi} \dot{e}_{\chi} \\
& +k_{p l} e_{l_{1}} \dot{e}_{l_{1}}+\left(m_{1}+m_{2}\right) g l_{1} \dot{\theta}_{1} s_{1} \\
& +m_{2} g l_{2} \dot{\theta}_{2} s_{2}+\left(m_{1}+m_{2}\right) g \dot{l}_{1}\left(1-c_{1}\right) \\
= & \dot{\mathbf{e}}^{\mathrm{T}}\left[\begin{array}{l}
\mathbf{U}+\mathbf{F}_{d}-\mathbf{M}(\mathbf{q}) \ddot{\mathbf{q}}_{d} \\
-\mathbf{G}(\mathbf{q})-\mathbf{V}(\mathbf{q}, \dot{\mathbf{q}}) \dot{\mathbf{q}}_{d}
\end{array}\right] \\
& +k_{p x} e_{\chi} \dot{e}_{\chi}+k_{p l} e_{l_{1}} \dot{e}_{l_{1}}+m_{2} g l_{2} \dot{\theta}_{2} s_{2} \\
= & \dot{e}_{\chi}\left[\begin{array}{l}
F_{x}-F_{r x}-d_{x} \dot{x}-k_{p x} e_{\chi} \\
-\left(M+m_{1}+m_{2}\right) \ddot{x}_{c}
\end{array}\right] \\
& +\dot{e}_{l_{1}}\left[\begin{array}{l}
F_{l}-d_{l} \dot{l}_{1}-k_{p l} e_{l_{1}} \\
+\left(m_{1}+m_{2}\right)\left(g-\ddot{x}_{c} s_{1}\right)
\end{array}\right] \\
& -\dot{\theta}_{1}\left[\ddot{x}_{c} l_{1}\left(m_{1}+m_{2}\right) c_{1}+d_{\theta_{1}} \dot{\theta}_{1}\right] \\
& -\dot{\theta}_{2}\left[\ddot{x}_{c} m_{2} l_{2} c_{2}+d_{\theta_{2}} \dot{\theta}_{2}\right]
\end{aligned}
$$


Property 2 is applied in the derivation of Equation (19), and its derivation principle is similar to equation (10).

Then, the proposed controller (15) is substituted into Equation (19), and it can be deduced that:

$$
\begin{aligned}
\dot{V}(t)= & -k_{d x} \dot{e}_{\chi}^{2}-k_{d l} \dot{e}_{l_{1}}^{2}-d_{\theta_{1}} \dot{\theta}_{1}^{2}-d_{\theta_{2}} \dot{\theta}_{2}^{2} \\
& -m_{2} l_{2} \dot{\theta}_{2} c_{2}\left(\begin{array}{c}
k_{1} \ddot{l}_{1} \int_{0}^{t} \theta_{1} d \tau+2 k_{1} \dot{l}_{1} \theta_{1} \\
+k_{1} l_{1} \dot{\theta}_{1}+k_{2} l_{2} \dot{\theta}_{2}-\ddot{x}_{r}
\end{array}\right) \\
& -\left(m_{1}+m_{2}\right)\left(\begin{array}{l}
k_{1} \ddot{l}_{1} \int_{0}^{t} \theta_{1} d \tau+2 k_{1} \dot{l}_{1} \theta_{1} \\
+k_{1} l_{1} \dot{\theta}_{1}+k_{2} l_{2} \dot{\theta}_{2}-\ddot{x}_{r}
\end{array}\right) \\
& \cdot l_{1} \dot{\theta}_{1} c_{1}
\end{aligned}
$$

In the actual transportation process, the swing angles should be limited within a small range to ensure security, which can be concluded as follows: $c_{i}=1$, $i=1,2$.

In view of the relationship between $k_{1}$ and $k_{2}$, and combined with $-\frac{\pi}{2}<\theta_{1}<\frac{\pi}{2}$ (see assumption 3 ), equation (20) is simplified and scaled. when $k_{1} \geq 0$, it can be seen that:

$$
\begin{aligned}
\dot{V}(t)= & -k_{d x} \dot{e}_{\chi}^{2}-k_{d l} \dot{e}_{l_{1}}^{2}-d_{\theta_{1}} \dot{\theta}_{1}^{2}-d_{\theta_{2}} \dot{\theta}_{2}^{2} \\
& -k_{1} l_{1}^{2}\left(m_{1}+m_{2}\right)\left[\dot{\theta}_{1}+\frac{m_{2} l_{2}}{\left(m_{1}+m_{2}\right) l_{1}} \dot{\theta}_{2}\right]^{2} \\
& -\left[\left(m_{1}+m_{2}\right) l_{1} \dot{\theta}_{1}+m_{2} l_{2} \dot{\theta}_{2}\right] \\
& \cdot\left(k_{1} \ddot{l}_{1} \int_{0}^{t} \theta_{1} d \tau+2 k_{1} \dot{l}_{1} \theta_{1}\right) \\
& -\left(m_{1}+m_{2}\right) l_{1} \dot{\theta}_{1} \ddot{x}_{r}-m_{2} l_{2} \dot{\theta}_{2} \ddot{x}_{r} \\
\leq & -k_{d x} \dot{e}_{\chi}^{2}-k_{d l} \dot{e}_{l_{1}}^{2}-d_{\theta_{1}} \dot{\theta}_{1}^{2}-d_{\theta_{2}} \dot{\theta}_{2}^{2} \\
& -\eta\left[\left(m_{1}+m_{2}\right) l_{1} \dot{\theta}_{1}+m_{2} l_{2} \dot{\theta}_{2}\right] \\
& +\left(m_{1}+m_{2}\right) l_{1}\left|\dot{\theta}_{1}\right|\left|\ddot{x}_{r}\right|+m_{2} l_{2}\left|\dot{\theta}_{2}\right|\left|\ddot{x}_{r}\right|
\end{aligned}
$$

Where $\eta$ stands for $k_{1} \ddot{l}_{1} \int_{0}^{t} \theta_{1} d \tau+2 k_{1} \dot{l}_{1} \theta_{1}$. According to $0 \leq\left|\ddot{l}_{1}\right| \leq \bar{a}_{l}$ (see assumption 2) and $-\frac{\pi}{2}<\theta_{1}<\frac{\pi}{2}$ (see assumption 4 ), it can be known that within finite time $t_{d}$ (the time required for the rope to reach the target position), there are:

$|\eta| \leq \frac{\pi}{2} k_{1} \bar{a}_{l} t_{d}+\pi k_{1} \bar{a}_{l} t_{d}=\frac{3}{2} \pi k_{1} \bar{a}_{l} t_{d}=|\eta|_{\max }$

According to the properties of the arithmetic-geometric mean inequality, the equation (21) is scaled as follows:

$$
\begin{aligned}
\dot{V}(t) \leq & -k_{d x} \dot{e}_{\chi}^{2}-k_{d l} \dot{e}_{l_{1}}^{2}-d_{\theta_{1}} \dot{\theta}_{1}^{2}-d_{\theta_{2}} \dot{\theta}_{2}^{2} \\
& +\frac{\left(m_{1}+m_{2}\right)^{2} l_{1}{ }^{2}}{2 d_{\theta_{1}}} \ddot{x}_{r}^{2}+\frac{1}{2} d_{\theta_{1}} \dot{\theta}_{1}^{2} \\
& +\frac{m_{2}^{2} l_{2}^{2}}{2 d_{\theta 2}} \ddot{x}_{r}^{2}+\frac{1}{2} d_{\theta_{2}} \dot{\theta}_{2}^{2} \\
& -\eta\left[\left(m_{1}+m_{2}\right) l_{1} \dot{\theta}_{1}+m_{2} l_{2} \dot{\theta}_{2}\right] \\
\leq & -k_{d x} \dot{e}_{\chi}^{2}-k_{d l} \dot{e}_{l_{1}}^{2}-\frac{1}{2} d_{\theta_{1}} \dot{\theta}_{1}^{2}-\frac{1}{2} d_{\theta_{2}} \dot{\theta}_{2}^{2} \\
& +\frac{\left(m_{1}+m_{2}\right)^{2} l_{1}^{2}}{2 d_{\theta_{1}}} \ddot{x}_{r}^{2}+\frac{m_{2}{ }^{2} l_{2}{ }^{2}}{2 d_{\theta 2}} \ddot{x}_{r}^{2} \\
& -\eta\left[\left(m_{1}+m_{2}\right) l_{1} \dot{\theta}_{1}+m_{2} l_{2} \dot{\theta}_{2}\right]
\end{aligned}
$$

$0<l_{1} \leq L$ is substituted into the first-order integral of equation (22), it can be inferred:

$$
\begin{aligned}
V(t) \leq & V(0)-k_{d x} \int_{0}^{t} \dot{e}_{\chi}^{2} d \tau-k_{d l} \int_{0}^{t} \dot{e}_{l_{1}}^{2} d \tau \\
& -\frac{d_{\theta_{1}}}{2} \int_{0}^{t} \dot{\theta}_{1}^{2} d \tau-\frac{d_{\theta_{2}}}{2} \int_{0}^{t} \dot{\theta}_{2}^{2} d \tau \\
& +\left[\frac{\left(m_{1}+m_{2}\right)^{2} L^{2}}{2 d_{\theta_{1}}}+\frac{m_{2}^{2} l_{2}^{2}}{2 d_{\theta_{2}}}\right] \int_{0}^{t} \ddot{x}_{r}^{2} d \tau \\
& -\eta\left[\left(m_{1}+m_{2}\right) L \int_{0}^{t} \dot{\theta}_{1} d \tau+m_{2} l_{2} \int_{0}^{t} \dot{\theta}_{2}^{2} d \tau\right]
\end{aligned}
$$

In view of the description of the expected trajectory $x_{r}$ in Principle 1, it can be deduced [26]:

$x_{r}, \dot{x}_{r}, \ddot{x}_{r}, x_{r}^{(3)} \in L_{\infty}$

According to principle 1, by using the method of integration by parts, it can be obtained [26]:

$$
\begin{aligned}
\int_{0}^{t} \ddot{x}_{r}^{2} d \tau & \leq \int_{0}^{\infty} \ddot{x}_{r}^{2} d \tau \\
& \leq\left.\dot{x}_{r} \ddot{x}_{r}\right|_{0} ^{\infty}-\int_{0}^{\infty} \dot{x}_{r} x_{r}{ }^{(3)} d \tau \\
& <2 v_{x \max } a_{x \max }+2 j_{x \max } p_{d x} \\
& \Rightarrow \ddot{x}_{r} \in L_{2} \cap L_{\infty}
\end{aligned}
$$

Combining equations (23)-(25), and in view of hypothesis 2, we can deduce:

$$
\begin{aligned}
V(t) \leq & V(0)+\left[\frac{\left(m_{1}+m_{2}\right)^{2} L^{2}}{d_{\theta_{1}}}+\frac{m_{2}^{2} l_{2}^{2}}{d_{\theta 2}}\right] \\
& \cdot\left(v_{x \max } a_{x \max }+j_{x \max } p_{d x}\right) \\
& +\frac{1}{2} \pi|\eta|_{\max }\left[\left(m_{1}+m_{2}\right) L+m_{2} l_{2}\right] \ll+\infty
\end{aligned}
$$

Based on equations (18), (23), (25)-(26), and combined with principle 1 , it can be deduced [27]:

$$
\begin{aligned}
V(t) \in L_{\infty} & \Rightarrow e_{\chi}, \dot{e}_{\chi}, e_{l_{1}}, \dot{e}_{l_{1}}, \dot{\theta}_{1}, \dot{\theta}_{2} \in L_{\infty} \\
& \Rightarrow x, l_{1}, \dot{x}, \dot{l}_{1}, F_{x}, F_{l}, F_{r x} \in L_{\infty}
\end{aligned}
$$

Equation (26) is substituted into Equation (23), and the shifting process is performed to obtain:

$$
\begin{aligned}
& \quad \frac{d_{\theta_{1}}}{2} \int_{0}^{t} \dot{\theta}_{1}^{2} d \tau+\frac{d_{\theta_{2}}}{2} \int_{0}^{t} \dot{\theta}_{2}^{2} d \tau \\
& +k_{d x} \int_{0}^{t} \dot{e}_{\chi}^{2} d \tau+k_{d l} \int_{0}^{t} \dot{e}_{l_{1}}^{2} d \tau \\
& \leq V(0)-V(t)+\left(v_{x \max } a_{x \max }+j_{x \max } p_{d x}\right) \\
& \quad \cdot\left[\frac{\left(m_{1}+m_{2}\right)^{2} L^{2}}{d_{\theta_{1}}}+\frac{m_{2}^{2} l_{2}{ }^{2}}{d_{\theta 2}}\right] \\
& \quad+\frac{1}{2} \pi|\eta|_{\max }\left[\left(m_{1}+m_{2}\right) L+m_{2} l_{2}\right] \ll+\infty \\
& \Rightarrow \dot{e}_{\chi}, \dot{e}_{l_{1}}, \dot{\theta}_{1}, \dot{\theta}_{2} \in L_{2} \cap L_{\infty}
\end{aligned}
$$

By combining the conclusions of Equations (25), (26)-(27) and substituting them into equations (1)-(4), the conclusions can be deduced as follows:

$$
\ddot{x}, \ddot{l}_{1}, \ddot{\theta}_{1}, \ddot{\theta}_{2}, \ddot{e}_{\chi}, \ddot{e}_{l_{1}} \in L_{\infty}
$$

By combining the conclusions of Equations (28)(29) and using the extended Barbarat theorem [27], it can be inferred that:

$\lim _{t \rightarrow \infty}\left[\begin{array}{llll}\dot{e}_{\chi}(t) & \dot{e}_{l_{1}}(t) & \dot{\theta}_{1}(t) & \dot{\theta}_{2}(t)\end{array}\right]^{\mathrm{T}}=\left[\begin{array}{llll}0 & 0 & 0 & 0\end{array}\right]^{\mathrm{T}}$ 
According to $\dot{e}_{l_{1}}(t)=\dot{l}_{1}(t)$ and equation $(30)$, it can be inferred that:

$\lim _{t \rightarrow \infty} i_{1}(t)=0$

In combination with $\lim _{t \rightarrow \infty} \ddot{x}_{r}(t)=0$ and Equations (30)-(31), the proposed controller (15) is substituted into equations (1)-(4). When $t \rightarrow \infty$, the simple equation holds as follows:

$$
\left\{\begin{array}{l}
\left(M+m_{1}+m_{2}\right) \ddot{e}_{\chi}+\left[\sin \theta_{1} \ddot{l}_{1}+l_{1} \ddot{\theta}_{1} \cos \theta_{1}\right] \\
\cdot\left(m_{1}+m_{2}\right)+m_{2} l_{2} \ddot{\theta}_{2} \cos \theta_{2}=-k_{p x} e_{\chi} \\
\left(m_{1}+m_{2}\right)\left[\sin \theta_{1} \ddot{e}_{\chi}+\ddot{l}_{1}-g \cos \theta_{1}+g\right] \\
+m_{2} l_{2} \sin \left(\theta_{1}-\theta_{2}\right) \ddot{\theta}_{2}=-k_{p l} e_{l_{1}} \\
\left(m_{1}+m_{2}\right)\left[\cos \theta_{1} \ddot{e}_{\chi}+l_{1} \ddot{\theta}_{1}+g \sin \theta_{1}\right] \\
+m_{2} l_{2} \cos \left(\theta_{1}-\theta_{2}\right) \ddot{\theta}_{2}=0 \\
\cos \theta_{2} \ddot{e}_{\chi}+\sin \left(\theta_{1}-\theta_{2}\right) \ddot{l}_{1}+l_{1} \cos \left(\theta_{1}-\theta_{2}\right) \\
\ddot{\theta}_{1}+l_{2} \ddot{\theta}_{2}+g \sin \theta_{2}=0
\end{array}\right.
$$

Where when $t \rightarrow \infty, \ddot{e}_{\chi}=\ddot{x}-\ddot{x}_{r}-k_{1} \dot{\theta}_{1}-k_{2} \dot{\theta}_{2}=\ddot{x}$. $g_{1}(t)=\ddot{x}=\ddot{e}_{\chi}, g_{2}(t)=\ddot{l}_{1}, g_{3}(t)=\ddot{\theta}_{1}, g_{4}(t)=\ddot{\theta}_{2}$ is defined and substituted into equation (32). Then, the first derivative of the above equation is obtained, and the conclusion of Equation (27) is substituted into this equation. when $t \rightarrow \infty$, it can be inferred that:

$\lim _{t \rightarrow \infty} \dot{g}_{1}(t), \lim _{t \rightarrow \infty} \dot{g}_{2}(t), \lim _{t \rightarrow \infty} \dot{g}_{3}(t), \lim _{t \rightarrow \infty} \dot{g}_{4}(t) \in L_{\infty}$

The following basic knowledge of trigonometric functions is used to derive the equation:

$\sin \theta_{i}, \cos \theta_{i}, \sin \left(\theta_{i}-\theta_{j}\right), \cos \left(\theta_{i}-\theta_{j}\right) \in L_{\infty}$

Combined with the conclusions of equations (30)(31), using the extended Barbalat theorem, it can be inferred that:

$$
\left\{\begin{array}{l}
\lim _{t \rightarrow \infty} \ddot{e}_{\chi}(t)=\lim _{t \rightarrow \infty} \ddot{x}(t)=0 \\
\lim _{t \rightarrow \infty} \ddot{\theta}_{1}(t)=\lim _{t \rightarrow \infty} \ddot{\theta}_{2}(t)=0 \\
\lim _{t \rightarrow \infty} \ddot{l}_{1}(t)=0
\end{array}\right.
$$

By substituting the conclusion of Equation (33) into Equation (32), we can get:

$\lim _{t \rightarrow \infty}\left[\begin{array}{llll}e_{\chi}(t) & e_{l_{1}}(t) & \theta_{1}(t) & \theta_{2}(t)\end{array}\right]^{\mathrm{T}}=\left[\begin{array}{llll}0 & 0 & 0 & 0\end{array}\right]^{\mathrm{T}}$

In view of $\dot{e}_{\chi}=\dot{x}-k_{1} \theta_{1}-k_{2} \theta_{2}-\dot{x}_{r}$, by combining the conclusions of equations (30), (34) and $\lim _{t \rightarrow \infty} \dot{x}_{r}(t)=0$, it can be deduced that:

$\lim _{t \rightarrow \infty} \dot{e}_{\chi}=\lim _{t \rightarrow \infty} \dot{x}=0$

In view of the conclusion in Equation (34), by combining equations (13)-(14) and principle 1 , it can be deduced that:

$\lim _{t \rightarrow \infty}\left[x(t) \quad l_{1}(t)\right]^{\mathrm{T}}=\left[\begin{array}{ll}p_{d x} & p_{d l}\end{array}\right]^{\mathrm{T}}$
In view of the conclusions of Equations (30)-(31) and (33)-(36), it can be concluded as follows:

$\lim _{t \rightarrow \infty}\left[\begin{array}{llllllll}x(t) & \dot{x}(t) & l_{1}(t) & i_{1}(t) & \theta_{1}(t) & \dot{\theta}_{1}(t) & \theta_{2}(t) & \dot{\theta}_{2}(t)\end{array}\right]^{\mathrm{T}}$ $=\left[\begin{array}{llllllll}p_{d x} & 0 & p_{d l} & 0 & 0 & 0 & 0 & 0\end{array}\right]^{\mathrm{T}}$

According to the above equations, it can be seen that the state quantities of the DPUGC linkage system gradually converge to the equilibrium state described in Theorem 1.

Therefore, Theorem 1 is proved.

\section{Simulation analysis and hardware experiment}

In this section, the lowering process of the load is considered. In MATLAB/Simulink, the performance of the proposed controller is tested through a great quantity of simulations and experiments. The linkage system parameters are shown in Table 1. Firstly, the proposed controller is compared with adaptive control method [5] and saturation control method [19] to prove the excellent performance of the proposed controller. Then, we test the robustness of the proposed controller by changing the load mass, target displacement, rope length, initial hook/load swing angle, and adding external disturbance. Finally, the actual crane system parameters are adopted to simulate the anti-swing effect of the proposed controller. The Saturation Method [19] does not consider the hoisting/lowering motion of the load. To facilitate comparison, a PD controller is applied to drive the lifting motion:

$F_{l}=-70 e_{l_{1}}-110 \dot{e}_{l_{1}}-\left(m_{1}+m_{2}\right) g$

The initial position $x(0)$, hook swing angle $\theta_{1}(0)$ and load swing angle $\theta_{2}(0)$ are set as 0 . The rope length$\mathrm{s}$ are set as $0.2 \mathrm{~m}$. The S-shaped tracking trajectory is selected as follows:

$x_{r}(t)=\frac{p_{d x}}{2}+\frac{k_{v}{ }^{2}}{4 k_{a}} \ln \left(\frac{\cosh \left(2 k_{a} t / k_{v}-\varepsilon\right)}{\cosh \left(2 k_{a} t / k_{v}-\varepsilon-2 p_{d x} k_{a} / k_{v}{ }^{2}\right)}\right)$

Where $k_{v}=0.9, k_{a}=0.5, \varepsilon=3$ refer to corresponding trajectory coefficients.

\subsection{Comparative simulation analysis}

For the sake of fairness, the gains of the proposed controller and two comparative controllers are fully debugged respectively, until a set of gains with the best performance are obtained. Specifically, the gains of the adaptive method [5] are carefully adjusted as $k_{\alpha 1}=2.8$, $k_{\beta 1}=9.2, k_{\alpha 2}=70, k_{\beta 2}=120, k=0.015, \lambda=0.01$, $L=2$. The gains of the saturation method [19] are fully debugged as $k_{p}=4, k_{d}=14.8, \alpha=-1$, and the gains of the proposed controller are selected as $k_{1}=5, k_{p x}=4$, $k_{d x}=50, k_{p l}=12, k_{d l}=18$. Then, the proposed con- 
Table 1 DPUGC linkage system parameters

\begin{tabular}{cccc}
\hline Name & Symbol & Numerical Value & Unit \\
\hline Trolley mass & $M$ & 10 & $\mathrm{~kg}$ \\
Hook mass & $m_{1}$ & 2 & $\mathrm{~kg}$ \\
Load mass & $m_{2}$ & 1 & $\mathrm{~kg}$ \\
Gravity acceleration & $g$ & 9.8 & $\mathrm{~m} / \mathrm{s}^{2}$ \\
Trolley target displacement & $p_{d x}$ & 2 & $\mathrm{~m}$ \\
Rope target length & $p_{d l}$ & 1 & $\mathrm{~m}$ \\
Static friction-related coefficient 1 & $f_{r 0 x}$ & 4.4 & $\mathrm{NA}$ \\
Static friction-related coefficient 2 & $\varepsilon_{x}$ & 0.01 & $\mathrm{NA}$ \\
Viscous friction-related parameter & $k_{r x}$ & 0.5 & $\mathrm{NA}$ \\
Trolley direction air resistance coefficient & $d_{x}$ & 0.3 & $\mathrm{NA}$ \\
Rope direction Mechanical friction coefficient & $d_{l}$ & 6.5 & $\mathrm{NA}$ \\
Air resistance coefficient of hook & $d_{\theta_{1}}$ & 0.06 & $\mathrm{NA}$ \\
Air resistance coefficient of load & $d_{\theta_{2}}$ & 0.06 & $\mathrm{NA}$ \\
\hline
\end{tabular}

Table 2 Performance index results for different controllers

\begin{tabular}{cccccccccc}
\hline Controllers & $p_{d x}$ & $p_{d l}$ & $\theta_{1 \mathrm{max}}$ & $\theta_{2 \mathrm{max}}$ & $\theta_{1 \mathrm{res}}$ & $\theta_{2 \mathrm{res}}$ & $t_{\mathrm{st}}$ & $t_{1 \mathrm{sp}}$ & $t_{2 \mathrm{sp}}$ \\
\hline Proposed controller & 2.002 & 1 & 2.46 & 2.53 & 0.28 & 0.28 & 7.7 & 7.4 & 7.4 \\
Adaptive method & 2 & 1 & 3.45 & 5.07 & 0.22 & 0.24 & 9.1 & 6.6 & 6.7 \\
Saturation method & 2.001 & 1 & 5.47 & 7.42 & 0.06 & 0.1 & 9.7 & 4.1 & 4.4 \\
\hline
\end{tabular}
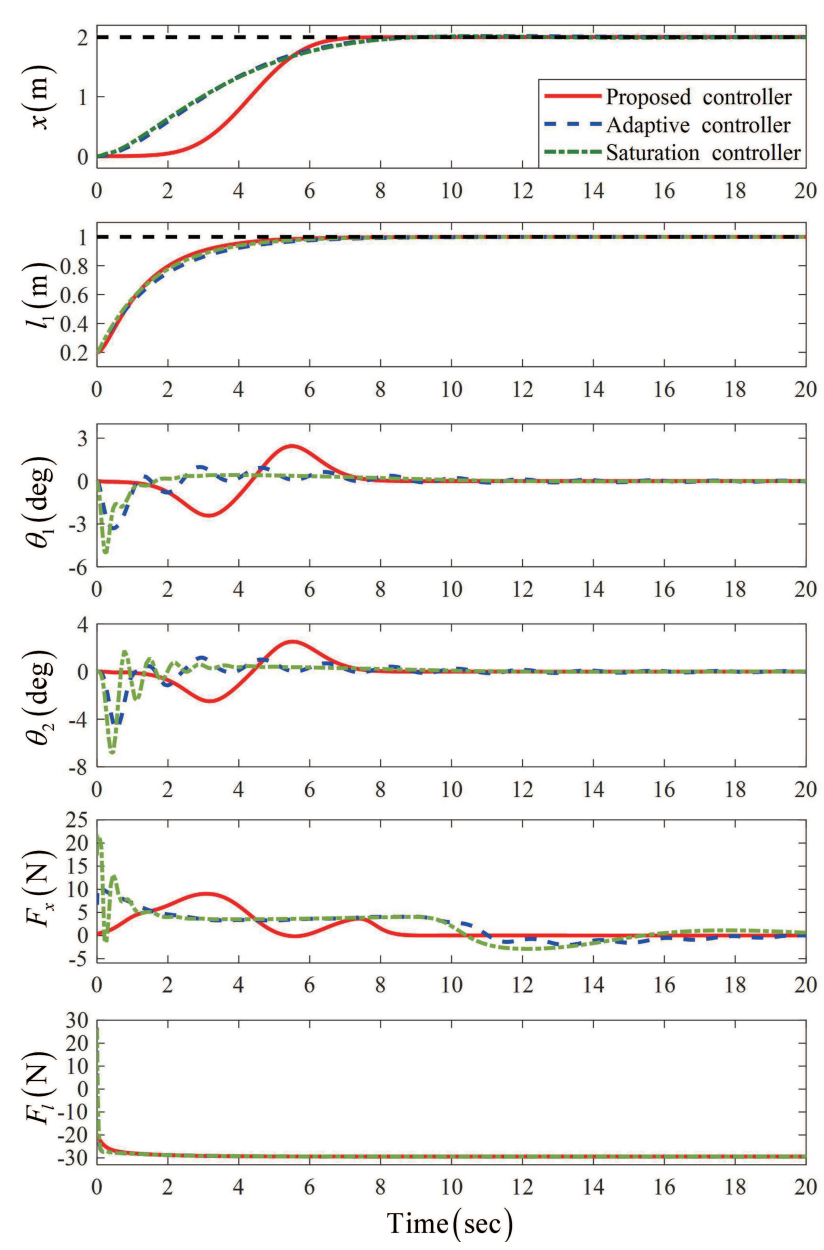

Fig. 3 Comparison simulation results troller, adaptive method [5] and saturation method [19] are simulated. The simulation results are shown in Figure 3 and the performance index results are shown in Table 2. Among them, the quantitative performance indexes exist to better compare the control performance of each controller quantitatively. Their indicators are described as follows:

1) $\theta_{i \max }, i=1,2$ is the maximum hook/load swing angle during the crane operation.

2) $\theta_{i \mathrm{res}}, i=1,2$ is the maximum hook/load swing angle after the crane stops running.

3) $t_{\mathrm{st}}$ is the time taken for the trolley to transport the load.

4) $t_{i \mathrm{sp}}, i=1,2$ is the time when the swing angles meet $\left|\theta_{i}(t) \leq 0.5^{\circ}\right|, \forall t \geq t_{i \mathrm{sp}}, i=1,2 . / /$

From the comparison results in Figure 3 and the performance indicators in Table 3 , we can see that the final positioning errors of these three controllers do not exceed $5 \mathrm{~mm}$. However, the transportation time of the proposed controller is obviously less than that of the other two controllers; under the control of the proposed controller, the starting/running process of the trolley is relatively stable, and the hook/load swing curves are relatively smooth. Moreover, it can better suppress the hook/load swing range, and there is no repeated swing phenomenon, which greatly improves the safety of gantry crane during operation. Although the hook/load swing is quickly suppressed under the control of the other two controllers, the swing hook/load angle changes drastically and the swings are too large when the trolley starts, which lack certain safety. Specifically, 
the proposed controller has the minimum transportation time $t_{\mathrm{st}}=7.7 \mathrm{~s}$, maximum hook swing $\theta_{1 \max }=$ $2.46 \mathrm{deg}$, and maximum load swing $\theta_{2 \max }=2.53 \mathrm{deg}$; The adaptive controller [5] has the minimum transportation time $t_{\mathrm{st}}=9.1 \mathrm{~s}$, maximum hook swing $\theta_{1 \max }=$ $3.45 \mathrm{deg}$, and maximum load swing $\theta_{2 \max }=5.07 \mathrm{deg}$; the saturation control method [19] has the minimum transportation time $t_{\mathrm{st}}=9.7 \mathrm{~s}$, maximum hook swing $\theta_{1 \max }=5.47 \mathrm{deg}$, and maximum load swing $\theta_{2 \max }=$ 7.42 deg. In summary, from the simulation results and the quantitative performance indicators, the proposed controller has an excellent effect on the swing suppression of the hook and load, and the transportation is fast, which significantly improve the transportation efficiency of the load.

\subsection{Robustness Verification}

In the actual working process of gantry crane, the load mass, load target displacement, initial hook/load swing angle are difficultly determined, and it is inevitable to be subject to external disturbance, which bring certain challenges to the control of the anti-swing controller undoubtedly. For verifying the robustness of the proposed controller, simulation tests are implemented for different load mass, load target displacement, hook/load initial swing angle and external disturbance respectively. Under the condition of constant control gains, simulation tests are divided into the following four cases:

Case 1 The robustness is tested for different load masses. Therefore, three different load masses are set as follows:
1) $m=1 \mathrm{~kg}$;
2) $m=6 \mathrm{~kg}$;
3) $m=0.3 \mathrm{~kg}$

From the simulation results in Figure 4, the proposed controller can also make the load reach the target position accurately under different load masses, and can effectively suppress and eliminate the hook/load swing. Specifically, under the condition of changing the load mass, the final positioning error of the trolley is no more than $2 \mathrm{~mm}$, the time to reach the target position is no more than $7.7 \mathrm{~s}$, and the maximum hook/load swing is no more than $2.53 \mathrm{deg}$. In addition, the starting/running process of the crane is relatively stable, and the hook/load swing will not produce violent repeated swings. Changing the load mass will not cause significant changes in each performance index. Therefore, the proposed controller has excellent robustness for different load masses.

Case 2 The robustness is tested for different load target displacements. Therefore, three different target displacements are set as follows:
1) $p_{d x}=2 \mathrm{~m}$;
2) $p_{d x}=1 \mathrm{~m}$
3) $p_{d x}=5 \mathrm{~m}$
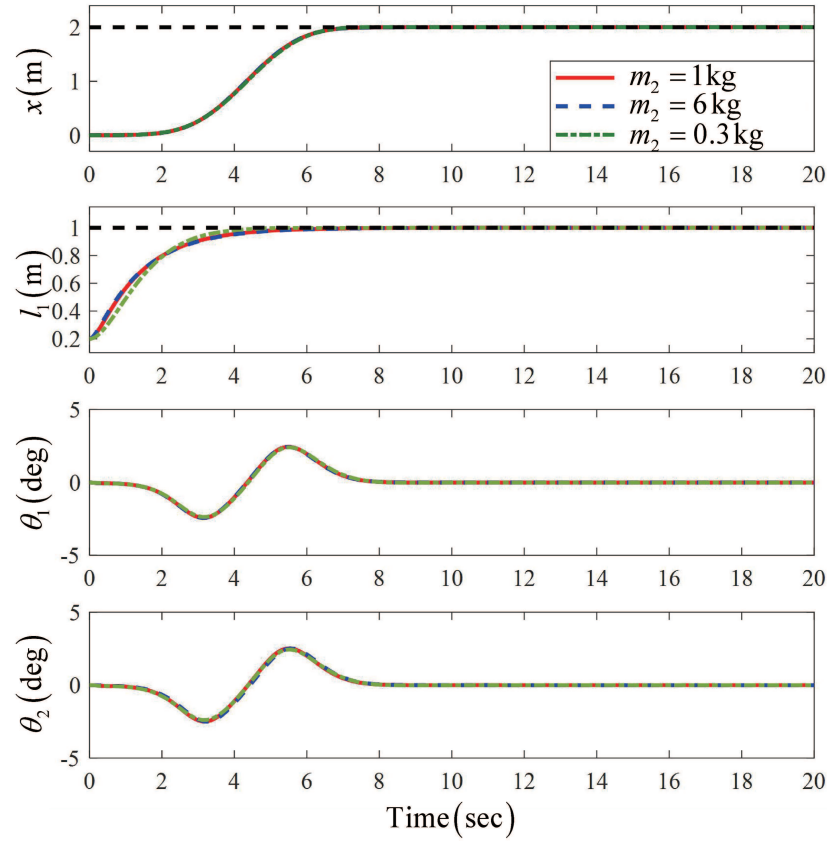

Fig. 4 Simulation results of the first case
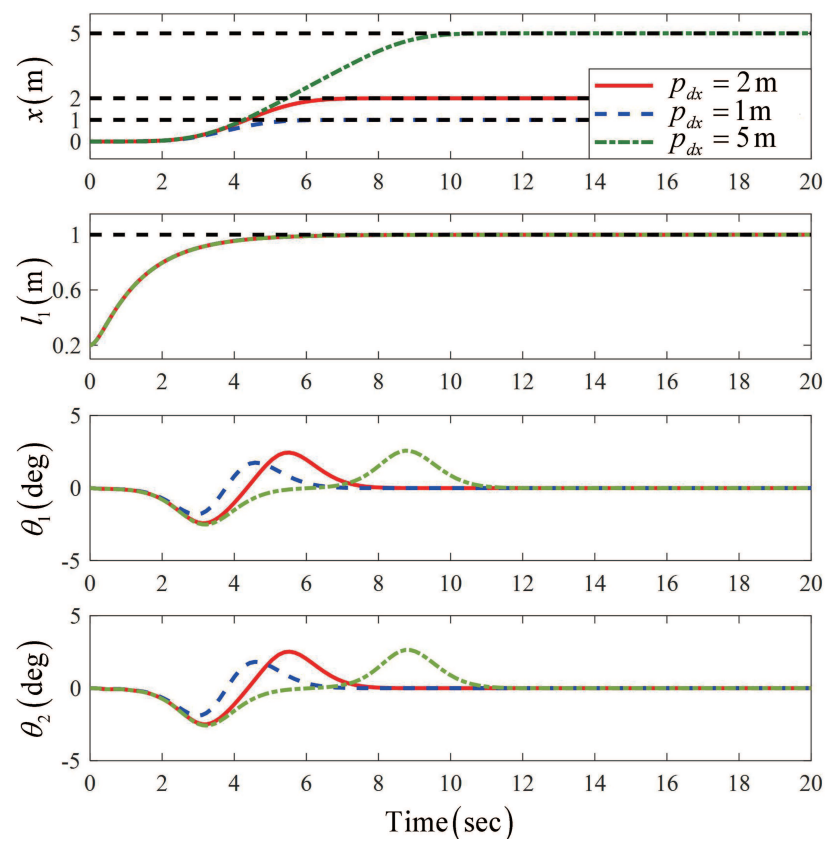

Fig. 5 Simulation results of the second case

From the simulation results in Figure 5, the proposed controller can accurately lift the load to different target positions. The time required for the trolley to reach the target position is $7.7 \mathrm{~s}, 6.6 \mathrm{~s}$ and $11 \mathrm{~s}$ respectively, and the final positioning error of the trolley is not more than $2 \mathrm{~mm}$, and the maximum hook/load swing angle is not more than $2.65 \mathrm{deg}$, the hook/load swing angle is quickly eliminated. Therefore, different target displacements have little effect on the hook/load 

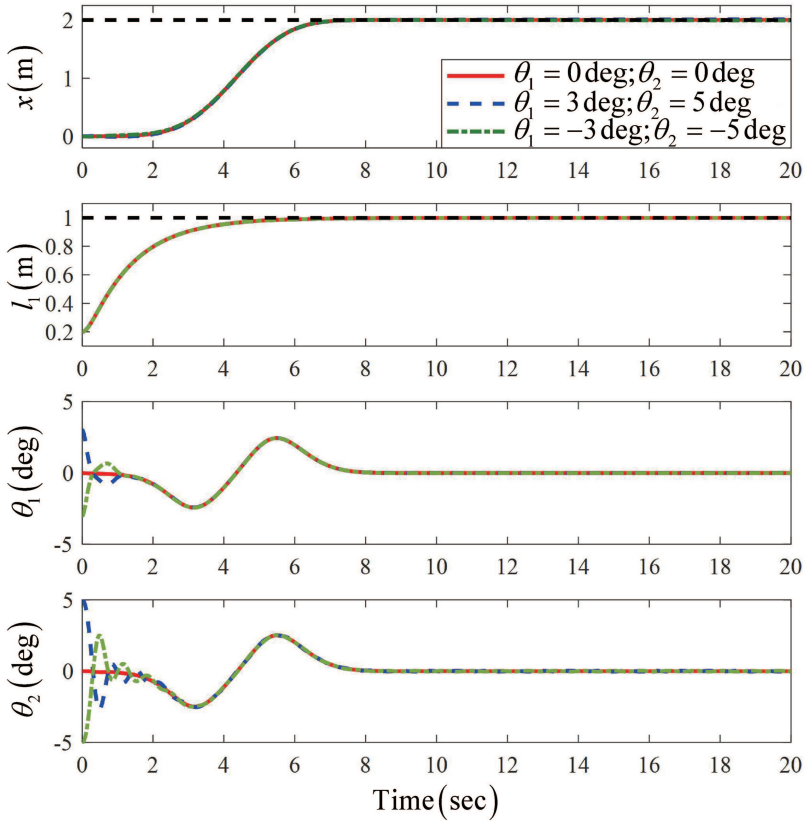

Fig. 6 Simulation results of the third case

swing, while the arrival time varies with distance, but the degree of change is relatively small.

Case 3 In this case, different initial hook swing angles and initial load swing angles are added to the linkage system, and the performance for different initial swings is tested. Because the proposed controller has excellent performance in suppressing and eliminating swing angles, the initial swing angles are set as follows:

1) $\theta_{1}=0 \mathrm{deg}, \theta_{2}=0 \mathrm{deg}$

2) $\theta_{1}=3 \mathrm{deg}, \theta_{2}=5 \mathrm{deg}$

3) $\theta_{1}=-3 \mathrm{deg}, \theta_{2}=-5 \mathrm{deg}$

From the simulation results in Figure 6, under the control of the proposed controller, the starting/running process of the trolley are relatively stable, and the existence of the initial hook/load swing angle has a negligible effect on the transportation efficiency of the trolley. Moreover, the positioning of the trolley is still very accurate; the initial hook/load swing angle is quickly eliminated, and their swings quickly tend to the swing states without the initial swing angles. Hence, the proposed controller has excellent performance for initial hook/load swing.

Case 4 For testing the robustness in response to external disturbance, a $5 \mathrm{deg}$ sinusoidal disturbance signal is added to the load swing angle in 10-12 s to simulate the external disturbance.

From the simulation results in Figure 7, the linkage system can eliminate the external disturbance and restore it to the equilibrium state quickly when it is subject to external disturbance. Specifically, the exter-
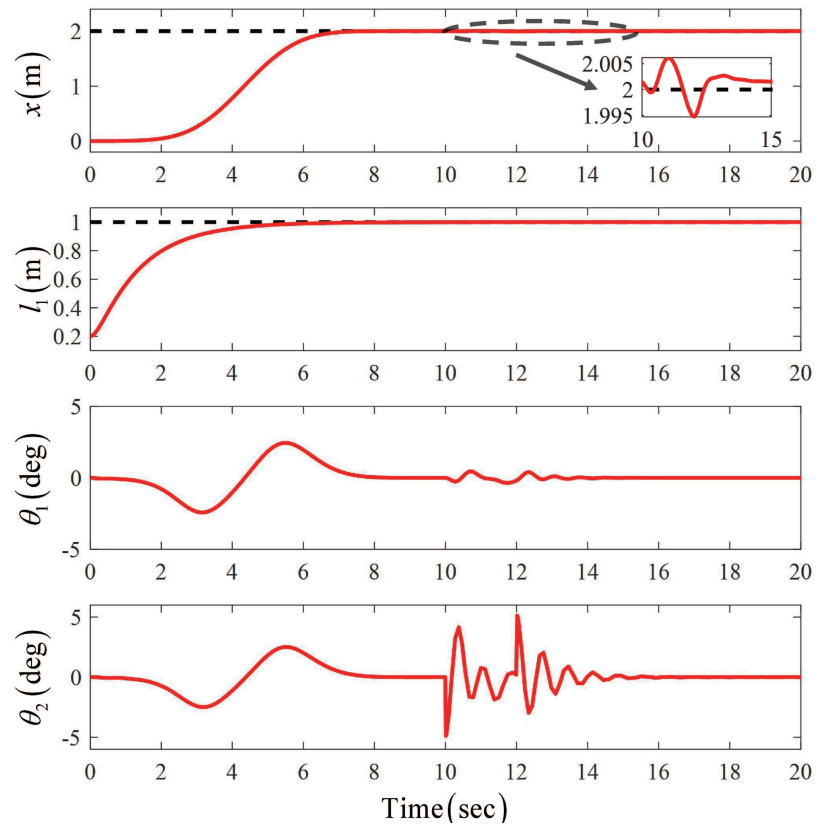

Fig. 7 Simulation results of the fourth case

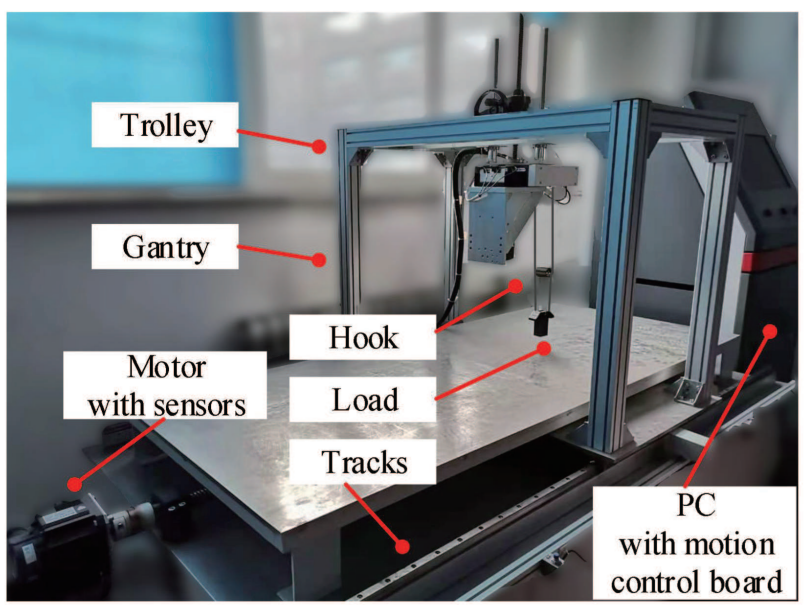

Fig. 8 Crane experimental system

nal disturbance has little impact on the positioning of the trolley, and the positioning error will not exceed 5 $\mathrm{mm}$, and it will quickly recover to the target position. When the load is disturbed by external disturbance, the impact on the hook is small, and the maximum swing angle is less than $0.48 \mathrm{deg}$. The hook/load swing angle will return to stable state within $2 \mathrm{~s}$ after the external disturbance disappears. Therefore, the proposed controller has good robustness against external disturbance. 


\subsection{Experimental analysis}

Although the proposed controller has excellent control performance in simulations, its control effect has not been verified in practice. Therefore, we build the crane experimental system as shown in Figure 8. The actual results of the proposed controller are obtained, and compared with the simulation results on the premise of keeping the control gains and crane system parameters unchanged. The trolley target displacement is set as 0.6 $\mathrm{m}$, and the rope target length is set as $0.8 \mathrm{~m}$.

Comparison results of the experiment and simulation are shown in Figure 9. Through experimental measurements, the actual transportation time of the trolley is similar to the simulation transportation time, but the actual maximum hook/load swing angle is larger than the simulation result; Due to the inevitable external interferences and hardware conditions in the experiment, the experimental curves have slight fluctuations, but the experimental curves is consistent with the overall trend of the simulation curves. Therefore, the proposed controller can still achieve excellent control effect in the experiment and has objective practical application.

\subsection{Simulation analysis for actual working condition}

Most existing controllers are designed and simulated for the crane experiment system, but few researches consider the application of actual crane system parameters to verify the effectiveness of the anti-swing controllers. It is inevitable that some anti-swing controllers can realize the anti-swing control of the experimental crane system, but it is difficult to realize the effective antiswing control of the actual crane system, and even difficult to be applied to the actual crane system. To solve this problem, a case of actual crane system parameters is selected to simulate and verify the anti-swing effect of the proposed controller.

The DPUGC system parameters are selected as follows:

$M=432 \mathrm{~kg}, m_{1}=257 \mathrm{~kg}, m_{2}=1750 \mathrm{~kg}, l_{1}(0)=2 \mathrm{~m}$, $l_{2}=1 \mathrm{~m}, p_{d x}=20 \mathrm{~m}, p_{d l}=6 \mathrm{~m}, k_{v}=2.4, k_{a}=1.2$, $\varepsilon=3.5$

The gains are selected as follows:

$k_{1}=5, k_{p x}=40, k_{d x}=500, k_{p l}=200, k_{d l}=1150$

From the simulation results in Figure 8, under the control of the proposed controller, the final positioning displacement $p_{d x}=20 \mathrm{~m}$, hoisting time $t_{\mathrm{st}}=24.4 \mathrm{~s}$, maximum hook swing angle $\theta_{1 \max }=2.99 \mathrm{deg}$ and maximum load swing angle $\theta_{2 \max }=3.01 \mathrm{deg}$ can be seen. Specifically, not only does the proposed controller ensure the accurate positioning of the trolley, but also it ensures the fast transportation of the crane; in the
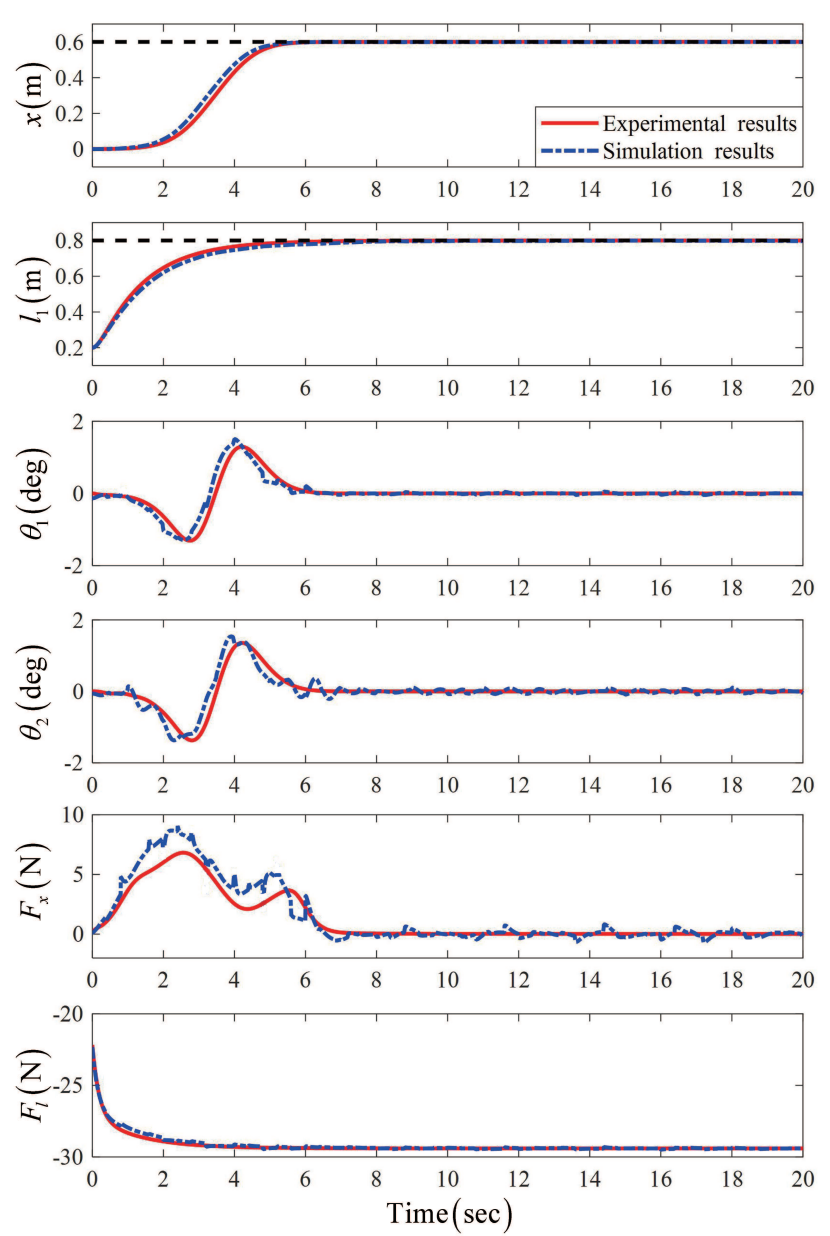

Fig. 9 Comparison results of the experiment and simulation

starting process, the trolley starts smoothly, and the initial hook/load swing is very small; in the working process, the hook/load swing curve is relatively smooth and the swing amplitude is small, moreover, the residual swing angles are quickly eliminated when the trolley stops running. The proposed controller still has better anti-swing control effect in the actual crane system, which lays a solid foundation for the application of the proposed controller, which is helpful to the development and implementation of crane automatic driving in actual working condition, and has great practical value.

\section{Conclusion}

This paper presents a nonlinear coupled tracking antiswing controller to solve the problems, such as low working efficiency, inaccurate positioning of the trolley, large hook/load swing, and poor anti-disturbance ability in the DPUGC linkage system during the working process. Compared with the existing adaptive controller and saturation controller, the proposed controller has more 

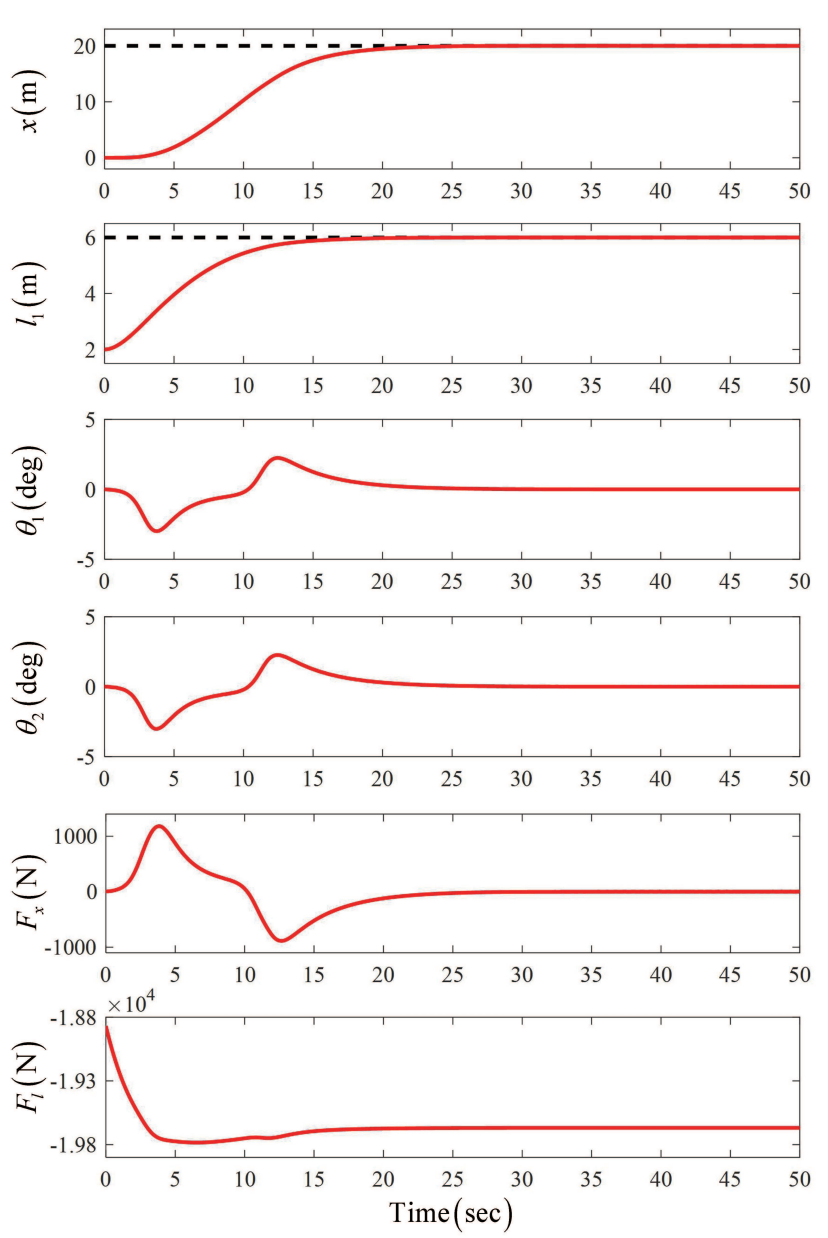

Fig. 10 Simulation results under actual working condition

outstanding control performance, saving at least $15 \%$ of the transport time; the hook/load swing range can be controlled within $3 \mathrm{deg}$, and the proposed controller can meet the requirements of efficient transportation and effective anti-swing of the crane; strong robustness can still be achieved under the condition of changing the system parameters and adding external disturbance; in addition, the proposed controller can still achieve excellent anti-swing control effect when the actual crane system parameters are adopted. In general, the proposed controller improves the transportation efficiency of gantry crane significantly, and can suppress and quickly eliminate the hook/load swing angle effectively, which is helpful for the research and development of the automatic driving of gantry crane, and has a great practical significance and good application prospects.

Acknowledgements This research was funded by the Science\&technology planning project of MOHURD 2019-K-080, and the National Natural Science Foundation of China under Grant 51905357, 52005352\&52075348.

\section{Conflict of interest}

The authors declare that they have no conflict of interest.

\section{References}

1. Shi, H., Li, G., Ma, X., Sun, J.: Research on nonlinear coupling anti-swing control method of double pendulum gantry crane based on improved energy. Symmetry. 11(12), 1511 (2019)

2. Sun, N., Zhang, J., Xin, X., Yang, T., Fang, Y.: Nonlinear output feedback control of flexible rope crane systems with state constraints. IEEE Access. 7, 136193C136202 (2019)

3. Shi, H., Li, G., Bai, X., Huang, J.: Research on nonlinear control method of underactuated gantry crane based on machine vision positioning. Symmetry. 11(8), 987 (2019)

4. Zhang, M., Ma, X., Rong, X., Sun, R., Tian, X.: Modeling and energy-based fuzzy controlling for underactuated overhead cranes with load transferring, lowering, and persistent external disturbances. Adv. Mech. Eng. 9(10), 1-13 (2017)

5. Lu, B., Fang, Y., Sun, N.: Enhanced-coupling adaptive control for double-pendulum overhead cranes with payload hoisting and lowering. Automatica. 101, 241-251 (2017)

6. Xia, X., Wu, Z.: Optimal motion planning for overhead cranes. IET Control Theory Appl. 8(17), 1833C1842 (2014)

7. Sun, N., Fang, Y., Chen, H.: A new anti-swing control method for underactuated cranes with unmodeled uncertainties: Theoretical design and hardware experiments. IEEE Trans. Ind. Electron. 62(1), 453C465 (2015)

8. Sun, N., Fang, Y., Chen, H., He, B.: Adaptive nonlinear crane control with load hoisting/lowering and unknown parameters: Design and experiments. IEEE/ASME Trans. Mechatronics. 20(5), 2107C2119 (2015)

9. Vazquez, Z., Collado, J., Fridman, L.: Control of a parametrically excited crane: A vector Lyapunovapproach. IEEE Trans. Control Syst. Technol. 21(6), 2332C2340 (2013)

10. Boschetti, G., Caracciolo, R., Richiedei, D., Trevisani, A.: A non-time based controller for load swing damping and path-tracking in robotic cranes. Intell. Robot. Syst. 76(2), 201C217 (2014)

11. Lee, L., Huang, C., Ku, S., Yang, Z., Chang, C.: Efficient visual feedback method to control a threedimensional overhead crane. IEEE Trans. Ind. Electron. 61(8), 4073C4083 (2014)

12. Masoud, Z., Alhazza, K., Abu-Nada, E., Majeed, M.: A hybrid command-shaper for double-pendulum overhead cranes. Vib. Control. 20(1), 24C37 (2014)

13. Tuan, L., Lee, S.: Sliding mode controls of doublependulum crane systems. Mech. Sci. Technol. 27(6), 1863C1873 (2013)

14. Sun, N., Fang, Y., Chen, H., Fu, Y.: Super-twisting-based anti-swing control for underactuated double pendulum cranes. IEEE/ASME Int. Conf. Adv. Intell. Mechatronics. pp. 749C754. (2015)

15. Qian, D., Tong, S., Yang, B., Lee, S.: Design of simultaneous input-shaping-based SIRMs fuzzy control for doublependulum-type overhead cranes. Bulletin of the Polish Academy of Sciences Technical Sciences. 63(4), 887-896 (2015) 
16. Chen, H., Fang, Y., Sun, N.: A swing constrained timeoptimal trajectory planning strategy for double pendulum crane systems. Nonlinear Dyn. 89(2), 1513C1524 (2017)

17. Sun, N., Wu, Y., Chen, H., Fang, Y.: An energy-optimal solution for transportation control of cranes with double pendulum dynamics: design and experiments. Mech. Syst. Signal Process. 102(MAR.1), 87C101 (2018)

18. Zhang, M., Ma, X., Chai, H., Rong, X., Tian, X., Li, Y.: A novel online motion planning method for double-pendulum overhead cranes. Nonlinear Dyn. 85(2), 1079C1090 (2016)

19. Sun, N., Fang, Y., Chen, H., Lu, B.: Amplitude-saturated nonlinear output feedback anti-swing control for underactuated cranes with double-pendulum cargo dynamics. IEEE Trans. Industr Inform. 64(3), 2135C2146 (2017)

20. Sun, N., Yang, T., Fang, Y.: Transportation control of double-pendulum cranes with a nonlinear quasi-PID scheme: design and experiments. IEEE Trans. Syst. Man Cybern. Syst. 49(7), 1408C1418 (2019)

21. Sun, N., Wu, Y., Fang, Y.: Nonlinear anti-swing control for crane systems with double-pendulum swing eects and uncertain parameters: design and experiments. IEEE Trans. Autom. Sci. Eng. 15, 1413C1422 (2018)
22. Zhang, M., Ma, X., Rong, X.: A novel energy-couplingbased control method for double-pendulum overhead cranes with initial control force constraint. Adv. Mech. Eng. 10(1), 168781401775221 (2018)

23. Zhang, M., Zhang, Y., Cheng, X.: An enhanced coupling PD with sliding mode control method for underactuated double-pendulum overhead crane systems. Int. J. Control Autom. Syst. 17, 1579C1588 (2019)

24. Tuan, L., Lee, S., Ko, D., Nho, L.: Combined control with sliding mode and partial feedback linearization for 3D overhead cranes. Int J Robust Nonlinear Control. 24(18), 3372C3386 (2014)

25. Chwa D. Sliding-mode-control-based robust finite-time antisway tracking control of 3 -D overhead cranes. IEEE Trans Ind Electron. 64(8), 6775C6784 (2017)

26. Sun, N., Fang, Y.: Nonlinear tracking control of underactuated cranes with load transferring and lowering: Theory and experimentation. Automatica. 50(9), 2350C2357 (2014)

27. Khalil, H.: Nonlinear Systems. 3rd ed. Englewood Cliffs: Prentice-Hall. (2002) 
Figures

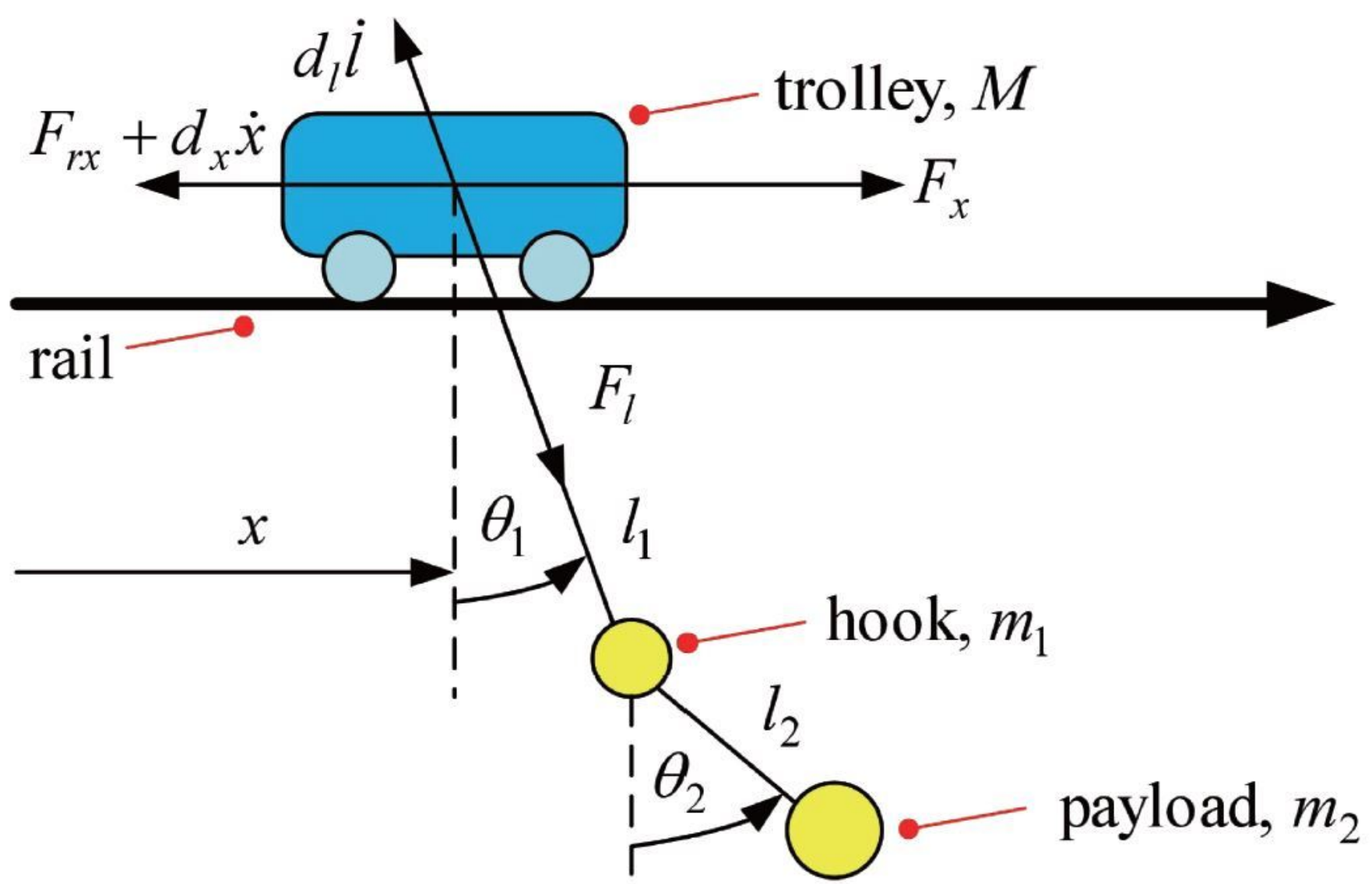

Figure 1

DPUGC linkage system

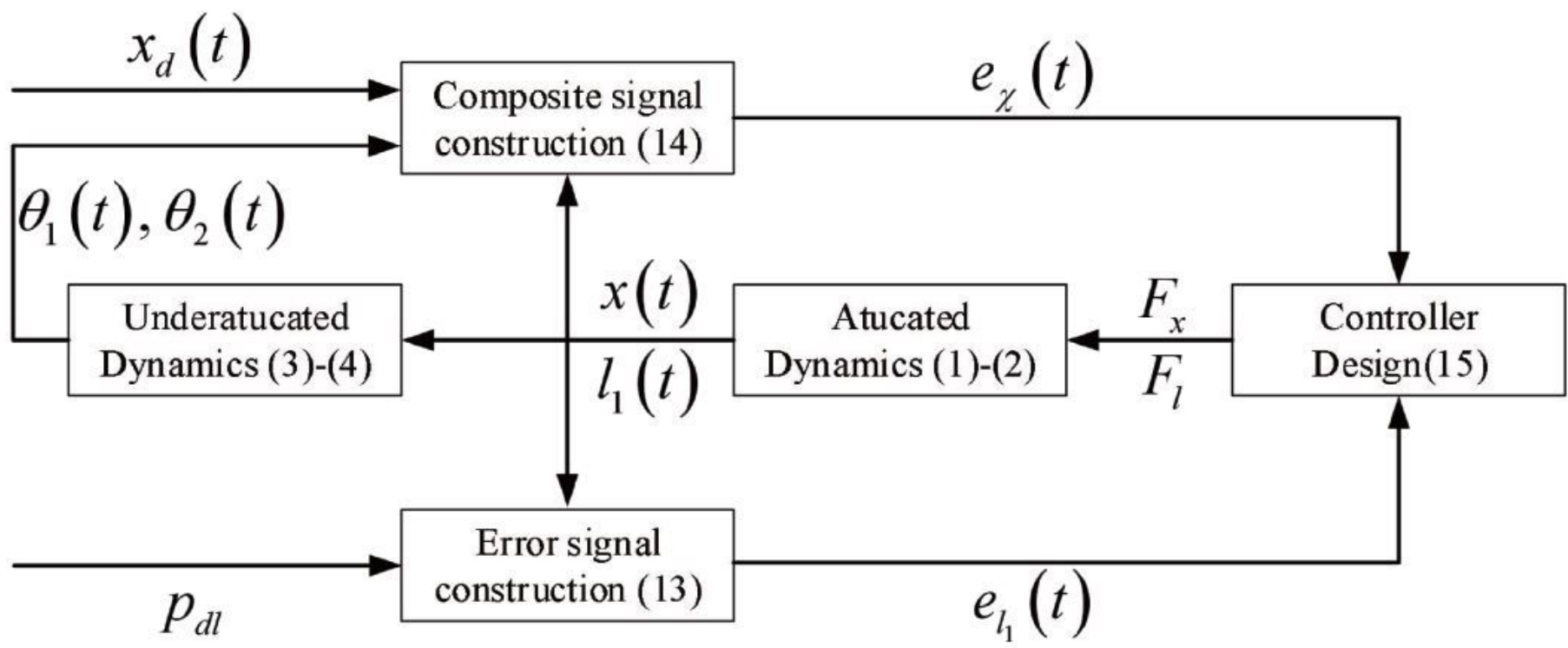


Figure 2

DPUGC control system
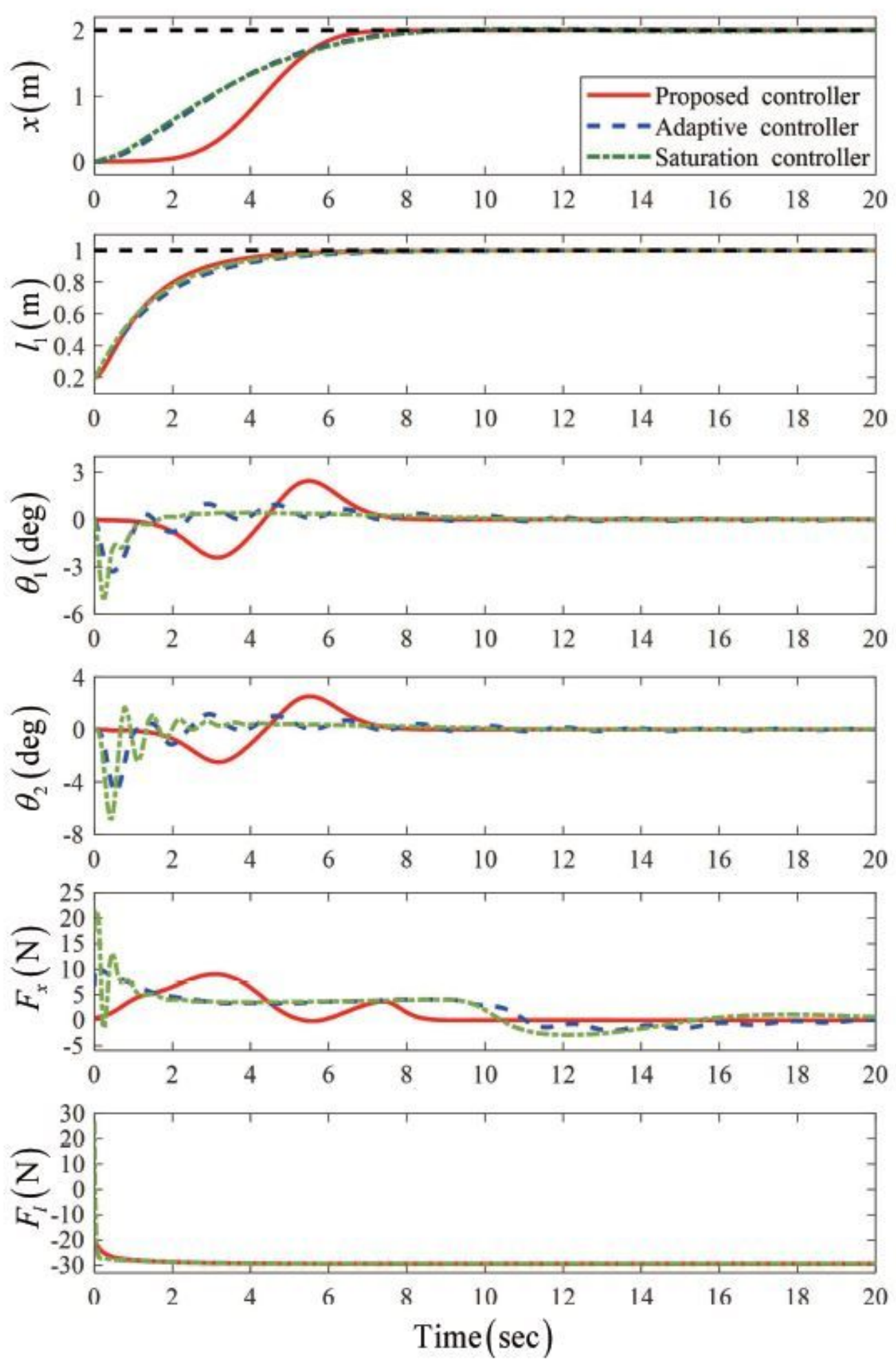

Figure 3

Comparison simulation results 

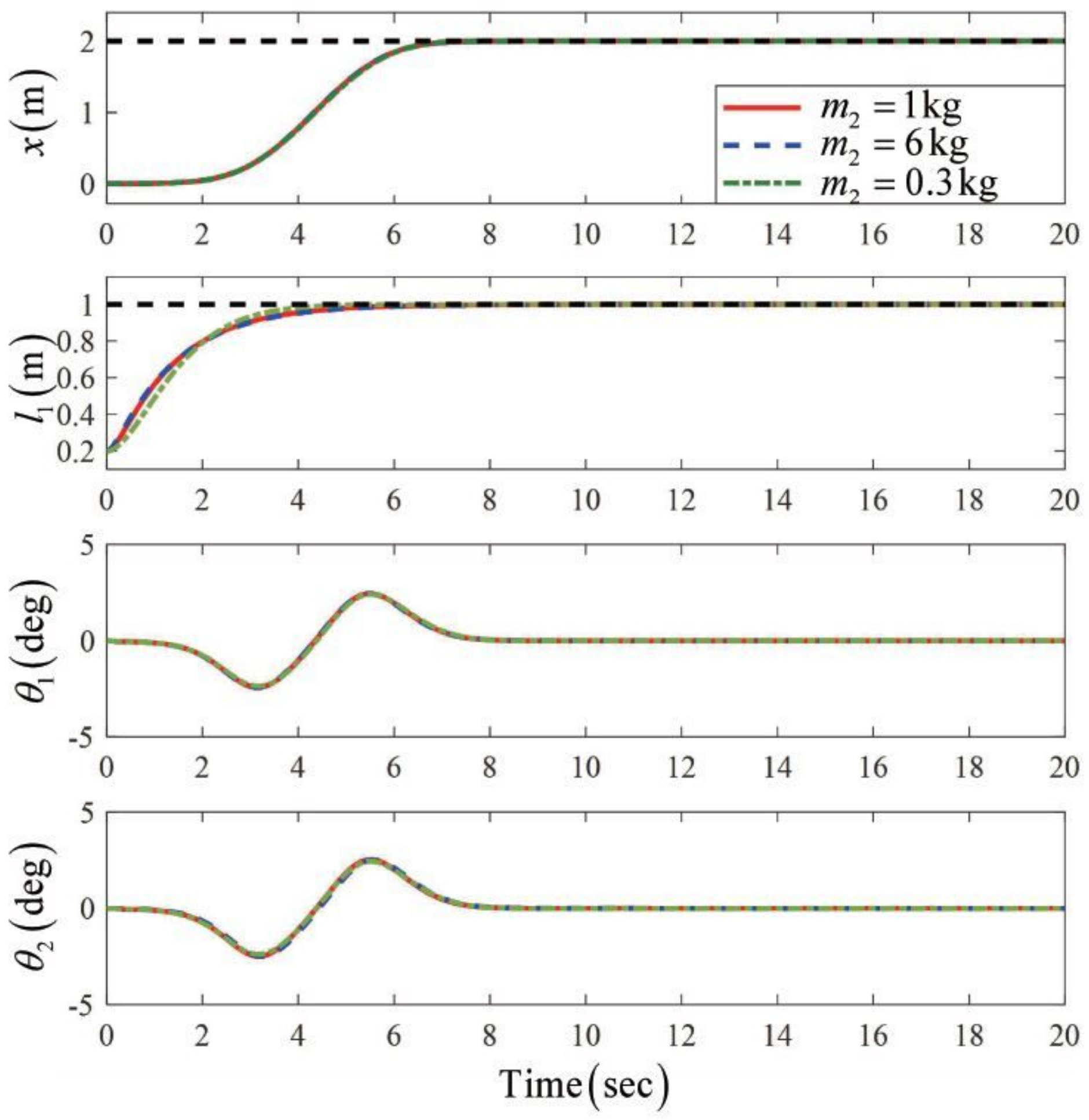

Figure 4

Simulation results of the rst case 

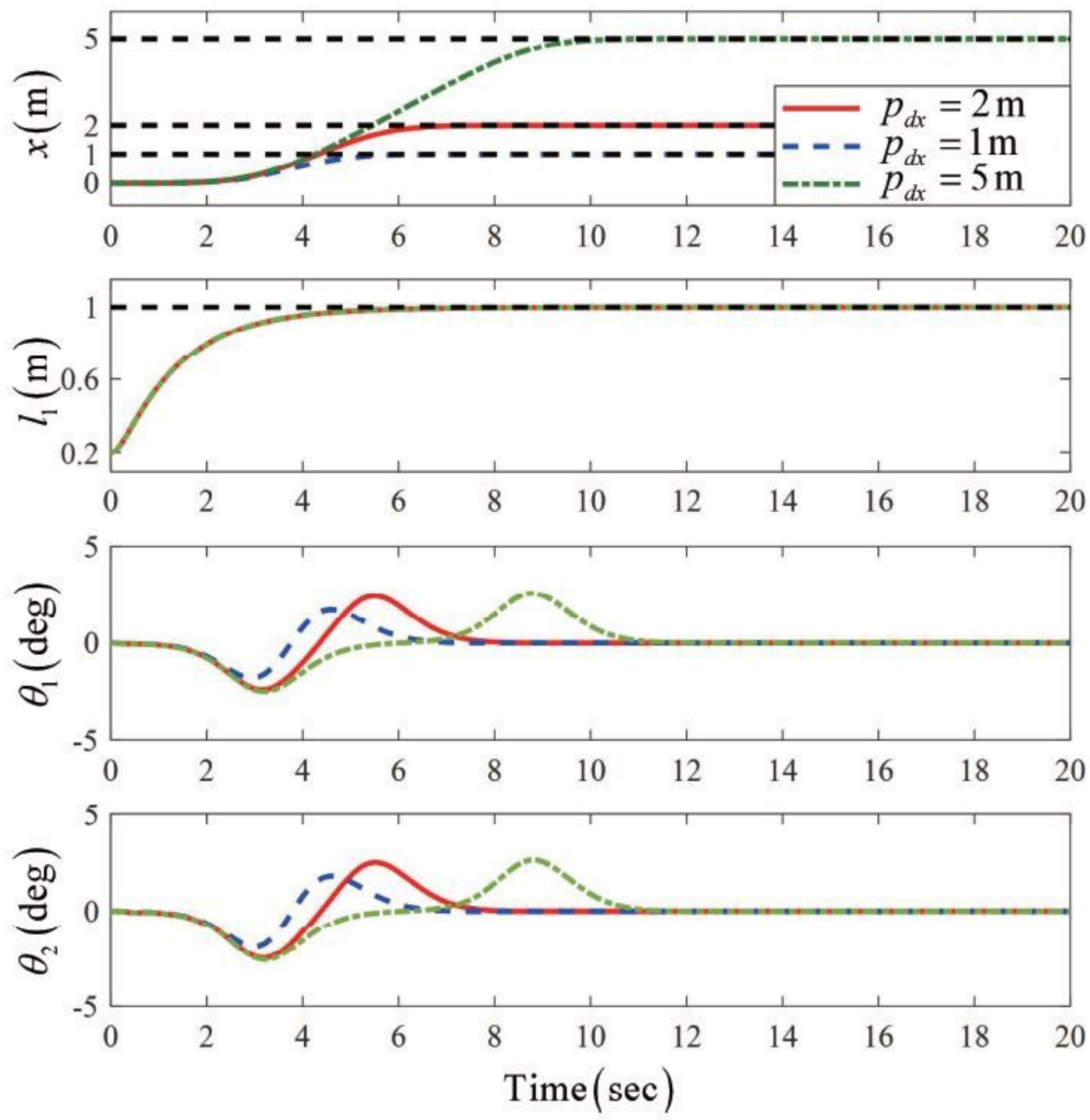

Figure 5

Simulation results of the second case 

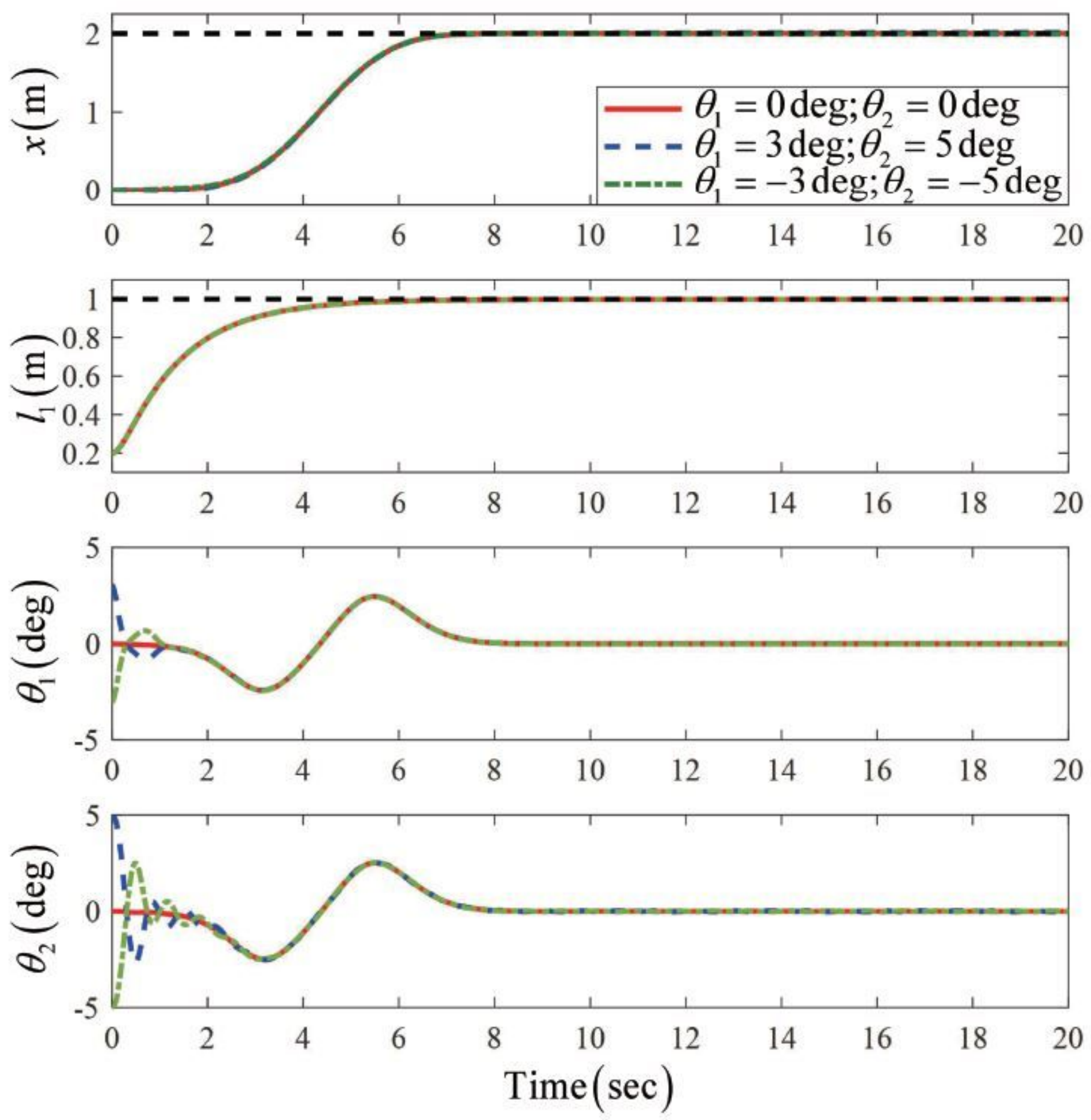

Figure 6

Simulation results of the third case 

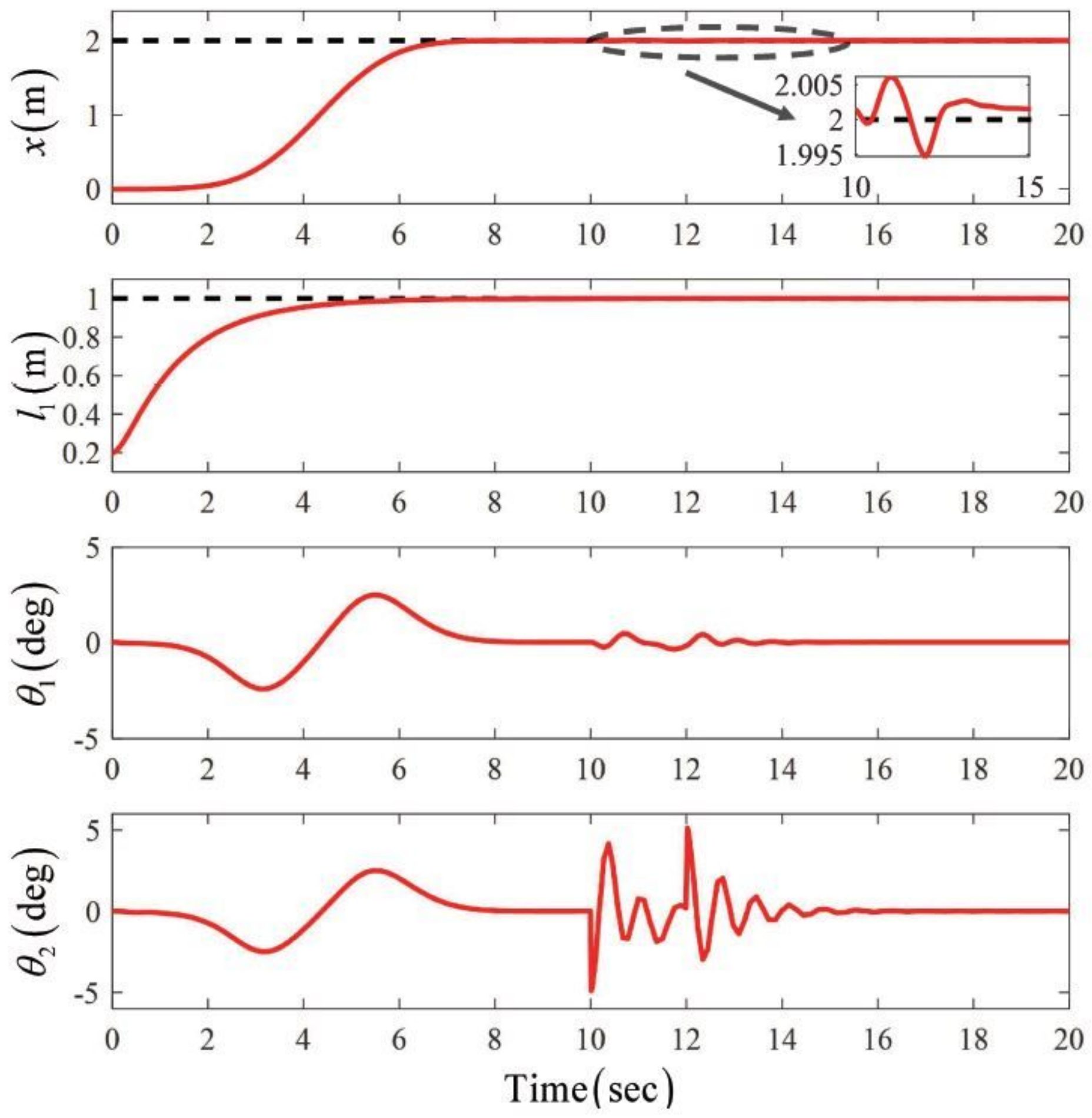

Figure 7

Simulation results of the fourth case 


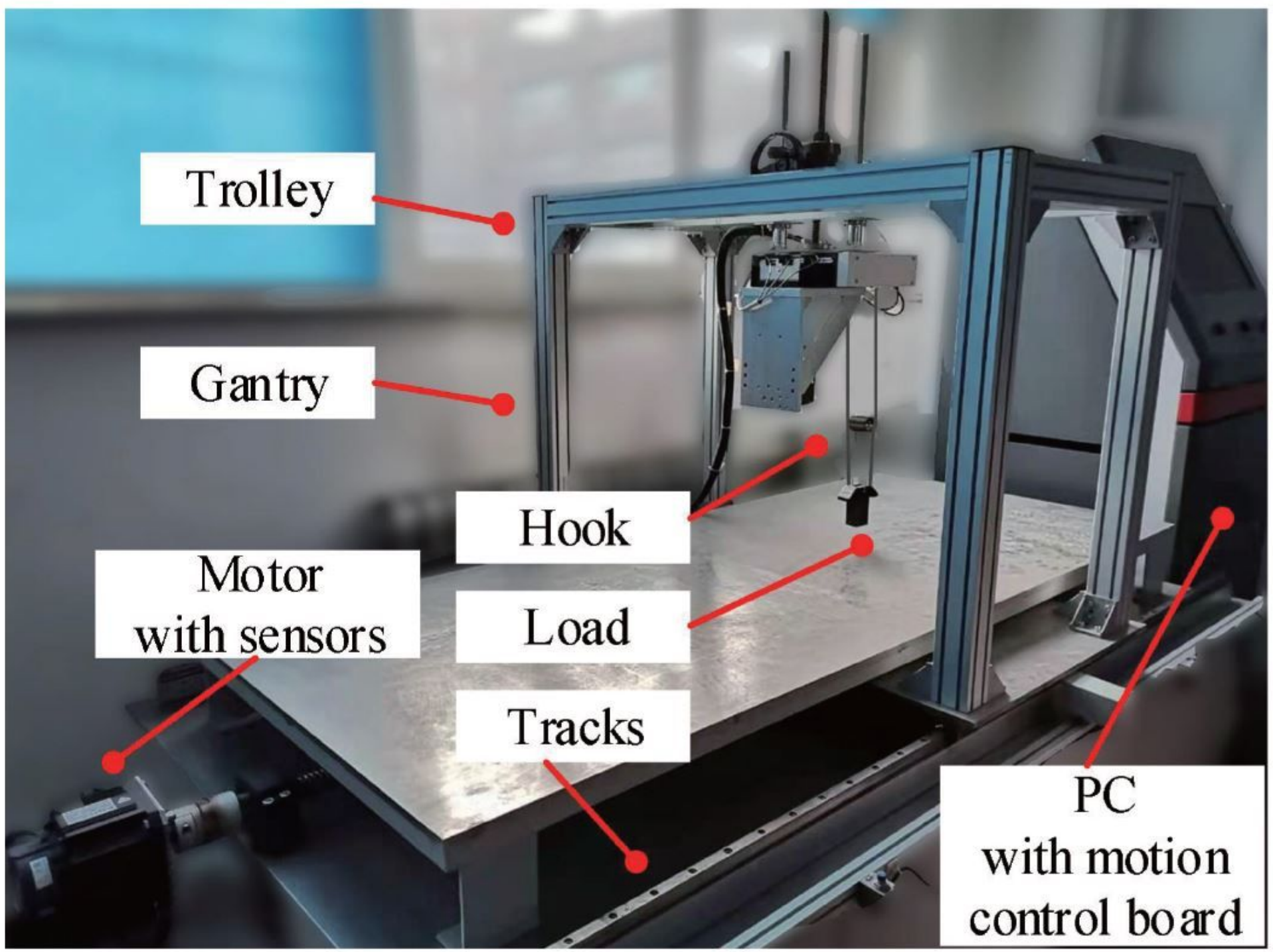

Figure 8

Crane experimental system 

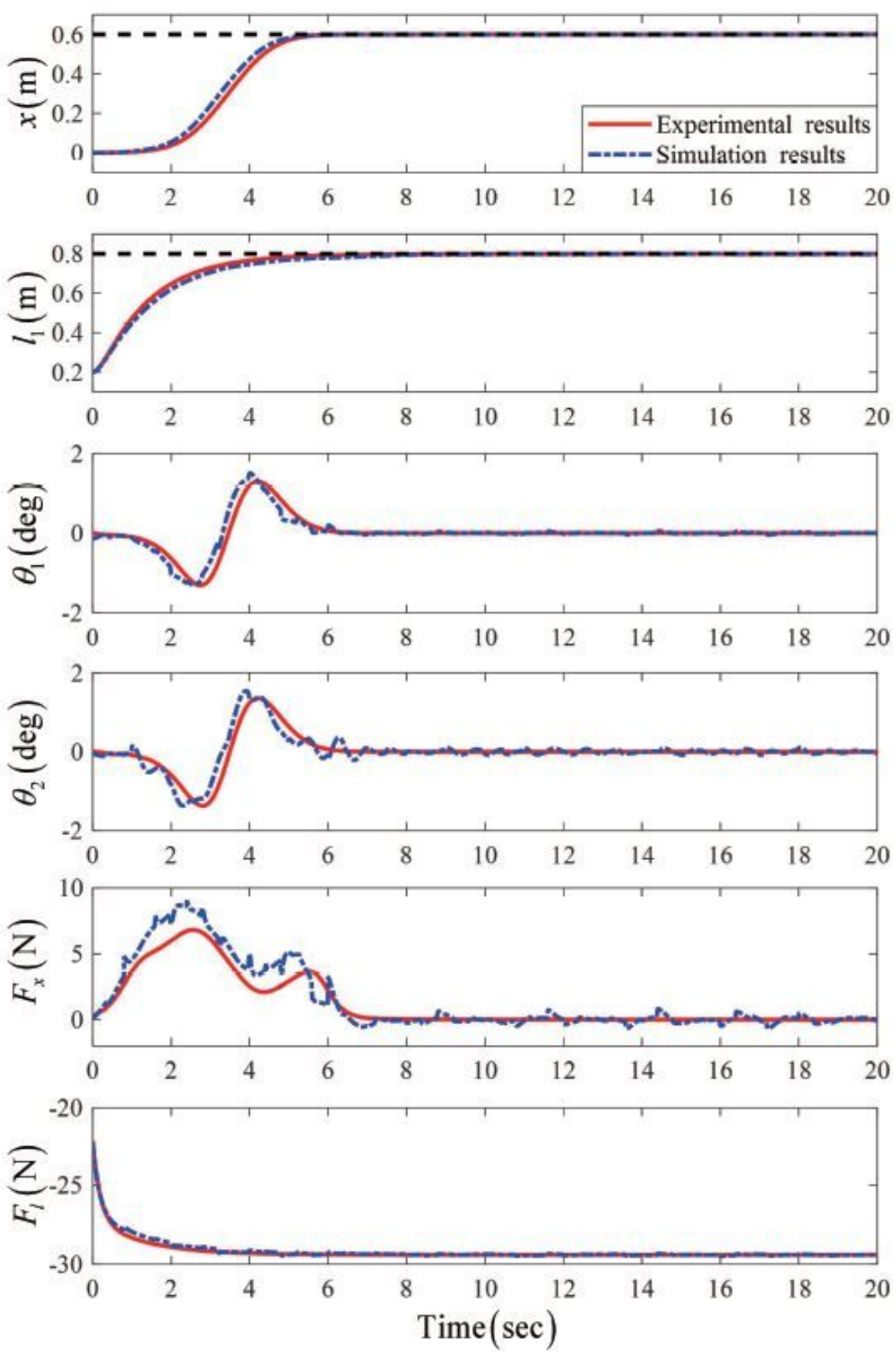

Figure 9

Comparison results of the experiment and simulation 

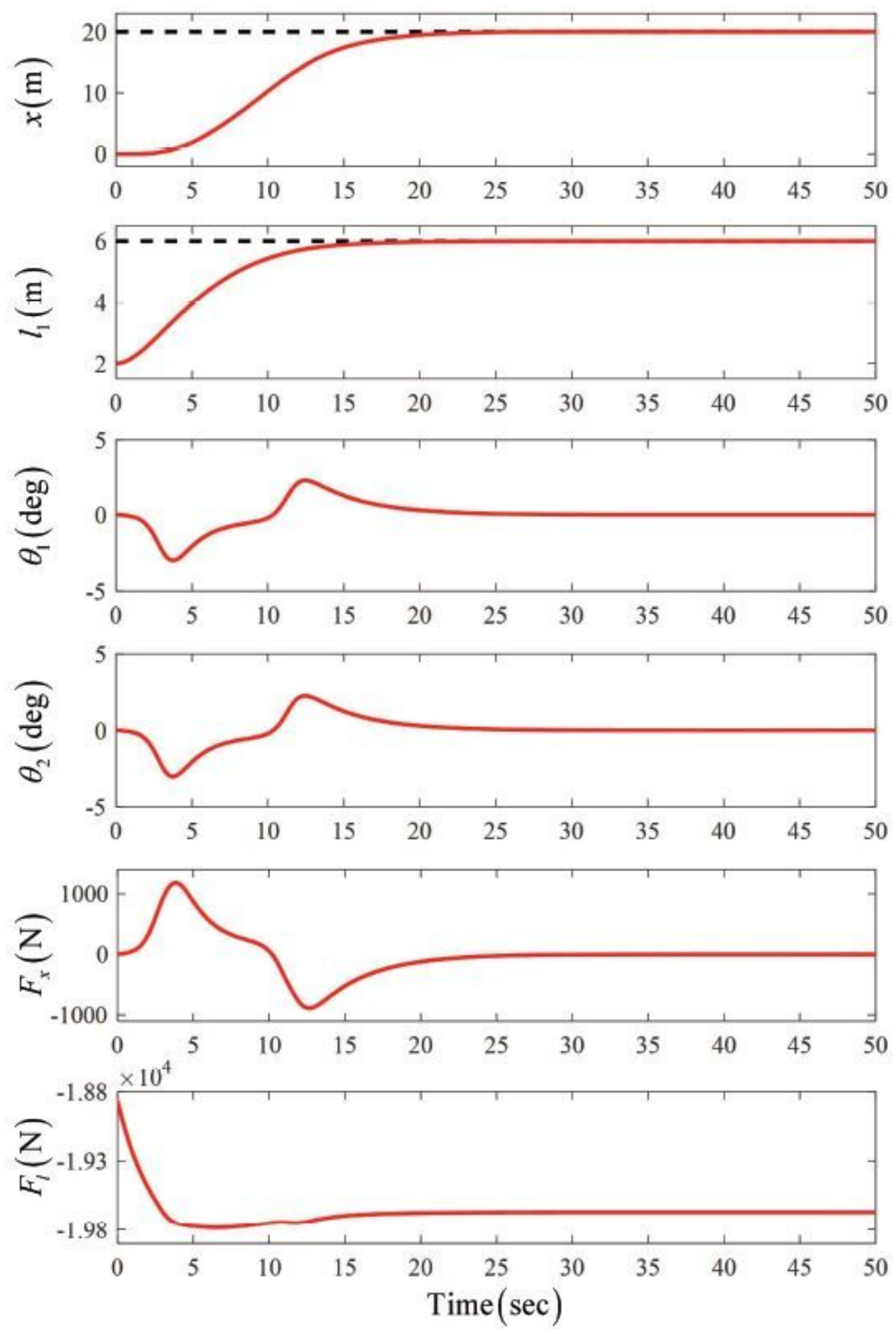

Figure 10

Simulation results under actual working condition 